\title{
Maternal obesity reduces oxidative capacity in fetal skeletal muscle of Japanese macaques
}

Carrie E. McCurdy, ${ }^{1,2}$ Simon Schenk, ${ }^{3}$ Byron Hetrick, ${ }^{1}$ Julie Houck, ${ }^{2}$ Brian G. Drew, ${ }^{4,5}$ Spencer Kaye, ${ }^{6}$ Melanie Lashbrook, ${ }^{6}$ Bryan C. Bergman, ${ }^{7}$ Diana L. Takahashi, ${ }^{8}$ Tyler A. Dean, ${ }^{8}$ Travis Nemkov, ${ }^{9}$ Ilya Gertsman, ${ }^{10}$ Kirk C. Hansen, ${ }^{9}$ Andrew Philp, ${ }^{11}$ Andrea L. Hevener, ${ }^{4}$ Adam J. Chicco, ${ }^{6}$ Kjersti M. Aagaard, ${ }^{12}$ Kevin L. Grove, ${ }^{8,13}$ and Jacob E. Friedman ${ }^{2}$

'Department of Human Physiology, University of Oregon, Eugene, Oregon, USA. ²Department of Pediatrics, University of Colorado Anschutz Medical Campus, Aurora, Colorado, USA. ${ }^{3}$ Department of Orthopaedic Surgery, University of California, San Diego, La Jolla, California, USA. ${ }^{4}$ David Geffen School of Medicine, Department of Medicine, Division of Endocrinology, Diabetes and Hypertension, University of California, Los Angeles, Los Angeles, California, USA. ${ }^{5}$ Diabetes and Dyslipidaemia Laboratory, Baker IDI Heart and Diabetes Institute, Prahran, Victoria, Australia. ${ }^{6}$ Departments of Health and Exercise Science and Biomedical Sciences, Colorado State University, Fort Collins, Colorado, USA. 'Division of Endocrinology, Metabolism, and Diabetes, Department of Medicine, University of Colorado Anschutz Medical Campus, Aurora, Colorado, USA. ${ }^{8}$ Division of Diabetes, Obesity and Metabolism, Oregon National Primate Research Center, Beaverton, Oregon, USA. 'Department of Biochemistry and Molecular Genetics, University of Colorado Anschutz Medical Campus, Aurora, Colorado, USA. ${ }^{10}$ Department of Pediatrics, University of California, San Diego, La Jolla, California, USA. ${ }^{11 S c h o o l ~ o f ~ S p o r t ~ E x e r c i s e ~ a n d ~ R e h a b i l i t a t i o n ~ S c i e n c e s, ~ U n i v e r s i t y ~ o f ~ B i r m i n g h a m, ~ B i r m i n g h a m, ~ B 15 ~ 2 T T, ~ U n i t e d ~ K i n g d o m . ~}$ ${ }^{12}$ Division of Maternal-Fetal Medicine, Department of Obstetrics and Gynecology, Baylor College of Medicine and Texas Children's Hospital, Houston, Texas, USA. ${ }^{13}$ Novo Nordisk Research Center, Seattle, Washington, USA.

Maternal obesity is proposed to alter the programming of metabolic systems in the offspring, increasing the risk for developing metabolic diseases; however, the cellular mechanisms remain poorly understood. Here, we used a nonhuman primate model to examine the impact of a maternal Western-style diet (WSD) alone, or in combination with obesity (Ob/WSD), on fetal skeletal muscle metabolism studied in the early third trimester. We find that fetal muscle responds to Ob/WSD by upregulating fatty acid metabolism, mitochondrial complex activity, and metabolic switches (CPT-1, PDK4) that promote lipid utilization over glucose oxidation. Ob/WSD fetuses also had reduced mitochondrial content, diminished oxidative capacity, and lower mitochondrial efficiency in muscle. The decrease in oxidative capacity and glucose metabolism was persistent in primary myotubes from Ob/WSD fetuses despite no additional lipid-induced stress. Switching obese mothers to a healthy diet prior to pregnancy did not improve fetal muscle mitochondrial function. Lastly, while maternal WSD alone led only to intermediary changes in fetal muscle metabolism, it was sufficient

Conflict of interest: B.C. Bergman is a coinventor on a patent application [205948-7027P1(00097)] titled Plasma Lipidomic Biomarkers of Ectopic Fat, filed 8/19/15. S. Schenk and A. Philp are coinventors on a patent application (PCT/US2014/011085) titled Method for Increasing Muscle Growth by Blocking Sirtuin Activity, filed 01/10/13. K.L. Grove is an employee of Novo Nordisk; however, no Novo Nordisk funds or resources were used in this study.

Submitted: February 2, 2016 Accepted: September 1, 2016 Published: October 6, 2016

Reference information: JCI Insight. 2016;1(16):e86612. doi:10.1172/jci.insight.86612. to increase oxidative damage and cellular stress. Our findings suggest that maternal obesity or WSD, alone or in combination, leads to programmed decreases in oxidative metabolism in offspring muscle. These alterations may have important implications for future health.

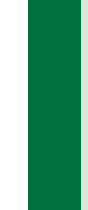

\section{Introduction}

In the United States, current estimates indicate that more than one half of women of reproductive age are overweight or obese $(1,2)$. Considering that the first 1,000 days of life is increasingly recognized as a critical period for establishing an individual's lifelong metabolic health $(3,4)$, it is perhaps not surprising that children from obese pregnancies have a greater risk than nonexposed children of developing obesity and components of metabolic syndrome in childhood $(5,6)$. In rodent models, adult offspring exposed to maternal obesity and/or a calorically dense diet during gestation and lactation also have a heightened disease risk when challenged by further environmental or dietary stress (7-9). Remarkably, however, the maternal-fetal stimuli and the mechanism by which an obesogenic fetal environment (i.e., defined here as 
nutrient excess combined with the inflammatory conditions and altered hormones associated with obesity) that leads to a reprogramming of metabolic pathways is still unknown. Increasing evidence points to a process of developmental programming, which alters the structure or function of a tissue due, in part, to epigenetic changes $(10,11)$.

Skeletal muscle has a primary role in maintaining glucose homeostasis, with reduced muscle insulin signaling significantly impacting metabolic health (12). In obese adults, muscle insulin resistance is linked to increased fatty acid availability and mitochondrial stress, which can increase cellular production of bioactive molecules including reactive oxygen species (ROS) (13), ceramides and diacylglycerides $(14,15)$, and mitochondria-derived acylcarnitines (16-18); all of these biomolecules can downregulate insulin signaling (19). In obese rodent and human skeletal muscle, directly relieving mitochondrial stress by increasing carbon efflux from mitochondria improves skeletal muscle oxidative metabolism and systemic glucose homeostasis $(20,21)$. Similarly, reducing fatty acid availability, independent of oxidative capacity, improves insulin sensitivity (22). Overall, these data and others indicate that mitochondrial bioenergetics and insulin action are interdependent and can be manipulated (e.g., increased or downregulated) to match cellular energy demand (23). While the impact of obesity on muscle substrate metabolism is well studied in the adult, less is known about how obesity during pregnancy effects fetal substrate metabolism, and more so, whether maternal obesity alters the metabolic programming linking energy sensing to mitochondrial metabolism and insulin sensitivity in the offspring.

Along these lines, metabolic derangements including elevated intramuscular diacylglycerides, impaired substrate oxidation, reduced mitochondrial number, and insulin resistance have been found in skeletal muscle from young, lean, adult offspring of parents with type 2 diabetes (24-28), suggesting that these metabolic pathways may be perturbed prior to the onset of obesity. In rodent models, skeletal muscle from adult offspring exposed to either a maternal high-fat/high-sucrose diet alone $(29,30)$ or combined with maternal obesity (31) had lower expression of genes related to mitochondrial biogenesis and oxidative metabolism as compared with unexposed offspring when challenged with a postnatal highfat diet. Moreover, a decrease in the F1 offspring muscle mitochondrial number, morphology, and electron transport system (ETS) complex abundance due to maternal high-fat/high-sucrose diet was shown to be persistent in females in the F2 and F3 generation, despite no further dietary challenge (30). These results suggest that the fetal mitochondrial system is highly susceptible to environmental stresses and that changes may be programmed into the offspring germline.

Several studies have also shown that maternal obesity can have a negative effect on mitochondrial function and substrate metabolism at the earliest stages of development in humans. Specifically, blastocysts from overweight and obese women (BMI > 25) compared with lean women undergoing in vitro fertilization were found to have abnormal metabolism with reduced glucose and pyruvate consumption, modified amino acid metabolism, and increased triglyceride (TAG) concentrations (32), providing evidence that mitochondrial adaptations can occur very early in development in response to maternal obesity. Additionally, these changes in substrate utilization occurred in parallel with poor functional outcomes including a greater likelihood of cell arrest (unable to form blastocysts) or smaller blastocysts that had a reduced total cell count. Adding to these findings, isolated oocytes from obese mice had increased mitochondria-derived oxidative stress (33), increased intracellular lipids, and reduced mitochondrial membrane potential (34). Together, these data show that substrate metabolism and mitochondrial function are susceptible to the maternal-fetal environment and may lead to persistent changes in the developing fetal tissue; however, to the best of our knowledge, no studies to date have examined the direct effect of maternal obesity on substrate metabolism in fetal or offspring skeletal muscle.

To address this gap in knowledge, in the present study, we investigated the specific impact of a typical Western-style diet (WSD) alone, or in combination with obesity, on muscle metabolism in fetal (gestational day 130 of 165) offspring in a nonhuman primate model. Our group has previously shown that initiating consumption of a higher fat, lower protein WSD early in the reproductive years in adult female Japanese macaques leads to a divergence in phenotypes such that a proportion of the adult females remain lean and insulin sensitive while the rest develop obesity $(35,36)$. We hypothesized that early fetal exposure to elevated fatty acids due to a chronic maternal consumption of a WSD would lead to reduced mitochondrial metabolism in fetal skeletal muscle and that these changes would be made worse by maternal obesity. 

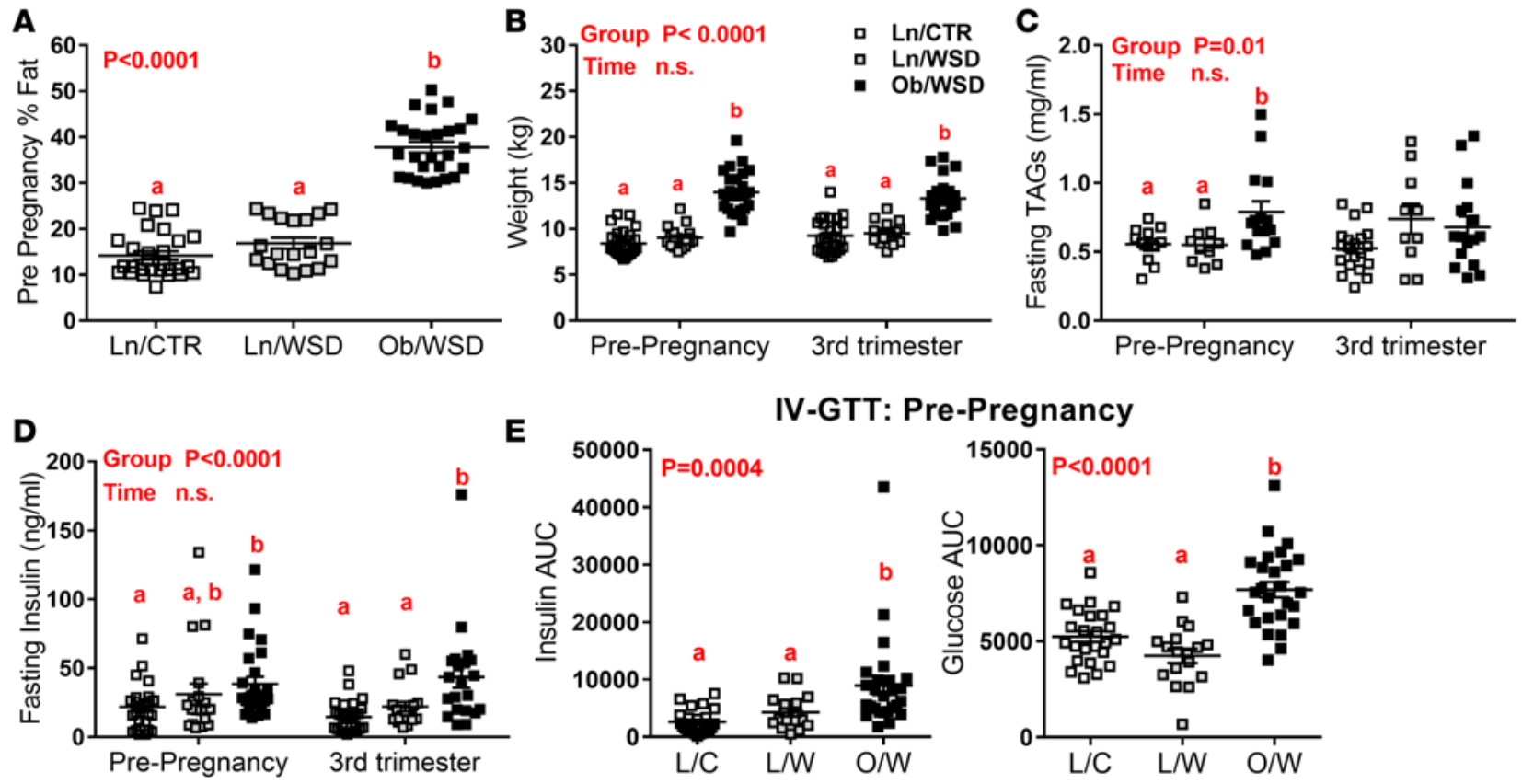

Figure 1. Chronic exposure to a Western-style diet (WSD) increases adiposity and insulin resistance in a subset of female Japanese macaques. (A) Adult female Japanese macaques fed a control diet (CTR or C) or Western-style diet (WSD or W) were grouped by percentage fat measured prior to pregnancy by DEXA into lean (Ln or L)/WSD ( $n=19 ; \leq 25 \%$ fat) to match Ln/CTR $(n=28)$ or obese (Ob or O)/WSD ( $n=27 ;>30 \%$ fat). (B) Body weight, (C) fasting plasma triglycerides (TAGs), and (D) insulin were measured in adult females prior to pregnancy and during the third trimester. (E) Insulin and glucose AUC was calculated from blood samples collected during an intravenous glucose tolerance test (IV-GTT) in anesthetized females before pregnancy in Ln/CTR $(n=24)$, Ln/WSD $(n=17)$, and Ob/WSD $(n=26)$. Data were analyzed by 1-way ANOVA (A and E) or 2-way ANOVA (pregnancy $\times$ group) with Tukey post-hoc tests. $P$ values for significant main effects are listed in each graph. Letters above groups are used to indicate significant post-hoc differences. Groups with the same letter are not significantly different from each other. Individual data points and the group mean \pm SEM are shown.

\section{Results}

WSD increases adiposity and insulin resistance in some, but not all, adult mothers. As previously reported (35), a subset of adult female Japanese macaques on a WSD do not gain a significant amount of weight, as compared with age- and parity-matched females fed a control chow diet (CTR). Fortuitously, this allows comparison of the effect of maternal WSD and obesity (Ob/WSD) with those of WSD and CTR without obesity (Ln/WSD and Ln/CTR, respectively) on fetal skeletal muscle outcomes; obese is defined as a nonpregnant body fat percentage of greater than 30\% and lean as less than 25\% (Figure 1A). As expected, prepregnancy and third trimester pregnancy body weight was significantly higher in Ob/WSD versus Ln/CTR and Ln/WSD (Figure 1B). Fasting TAGs were 45\% higher in nonpregnant obese females compared with lean females (Figure 1C); they were less pronounced at the third trimester time point but trended higher in both maternal groups on the WSD versus CTR. Fasting insulin, but not glucose, was higher in the $\mathrm{Ob} /$ WSD versus $\mathrm{Ln} / \mathrm{CTR}$ in prepregnancy females and during the third trimester (Figure 1D and Supplemental Figure 1A; supplemental material available online with this article; doi:10.1172/jci.insight.86612DS1). There was a marked difference in insulin sensitivity between Ob/WSD and Ln/WSD. The insulin response during an i.v. glucose tolerance test (GTT) was greater in Ob/WSD compared with Ln/CTR and Ln/ WSD, both prepregnancy (Figure 1E) and during the third trimester (Supplemental Figure 1B); the glucose response was also higher in the prepregnancy test (Figure 1E). Despite significant differences in maternal adiposity and insulin sensitivity, there was no effect of maternal obesity or diet on third trimester fetal weight, or fetal insulin and glucose concentrations (Supplemental Figure 1, C-E).

Maternal diet and obesity upregulate markers of lipid metabolism in fetal skeletal muscle. In male and female $\mathrm{Ob} / \mathrm{WSD}$ offspring, the intramuscular TAG concentration was $\sim 50 \%$ lower in Ob/WSD compared with Ln/CTR (Figure 2A). In contrast, fatty acid (FA) concentrations were significantly higher in Ob/WSD muscle (Figure 2B). Additionally, muscle from male offspring in all groups exhibited higher FA concentrations than females. Muscle phospholipids (PLs) were also increased in Ln/WSD and Ob/WSD compared with Ln/CTR (Figure 2C). Protein abundance of carnitine palmitoyltransferase $1 \beta$ (CPT-1 $\beta$ ), the 
A

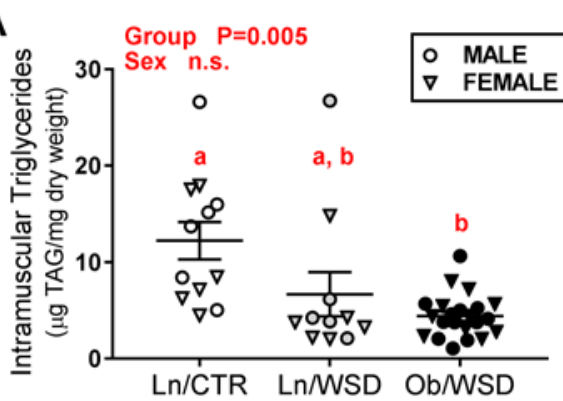

$B$

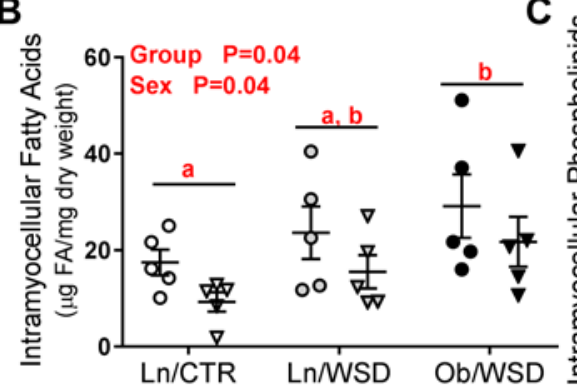

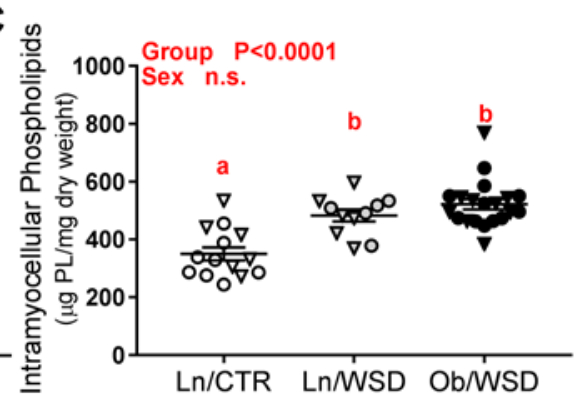

D

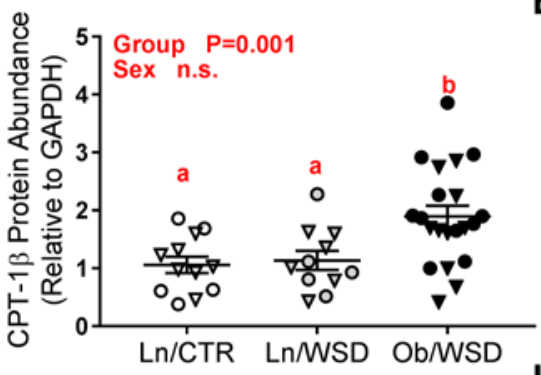

G
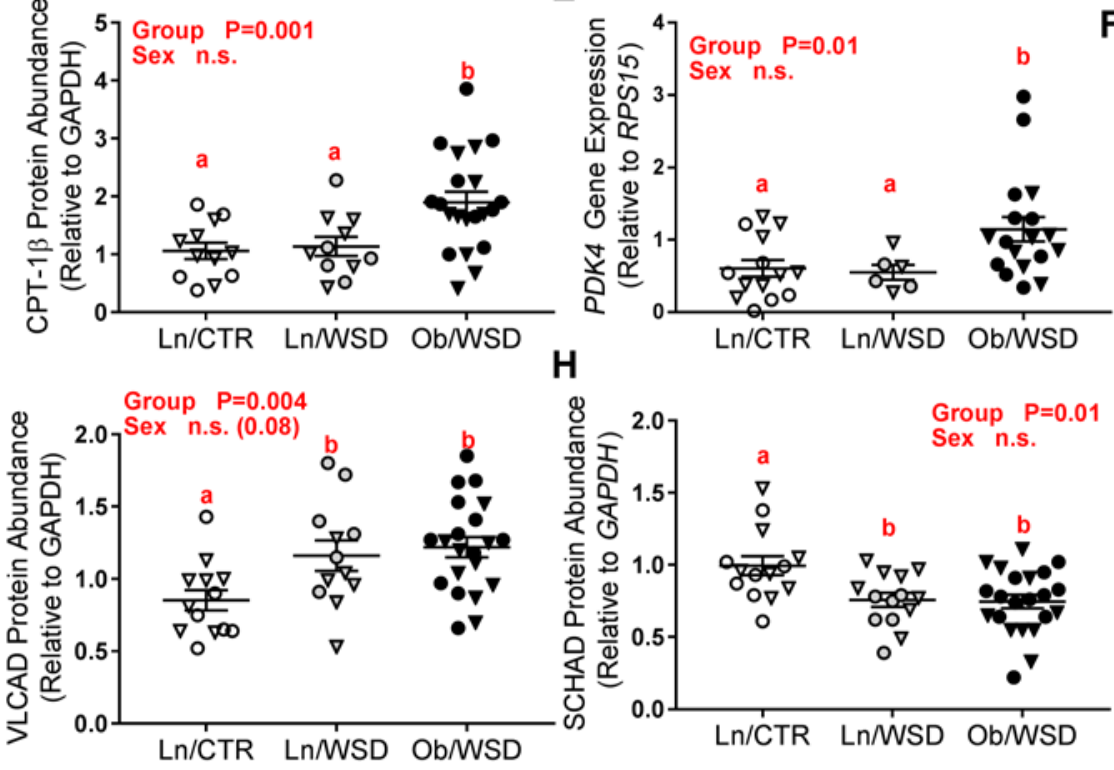

\section{H}

$\mathbf{F}$

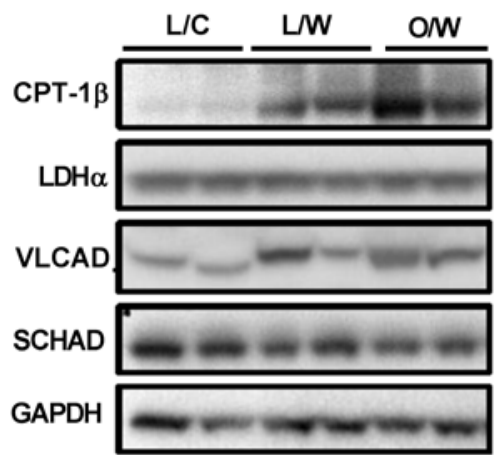

I

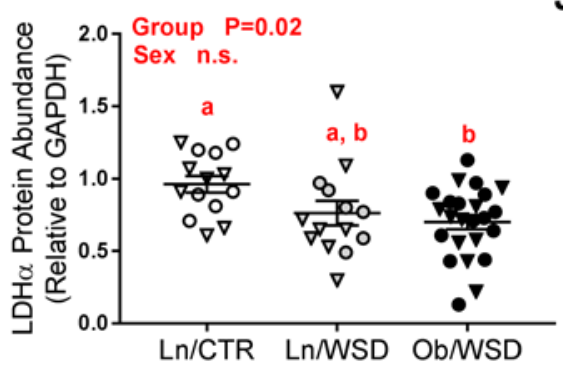

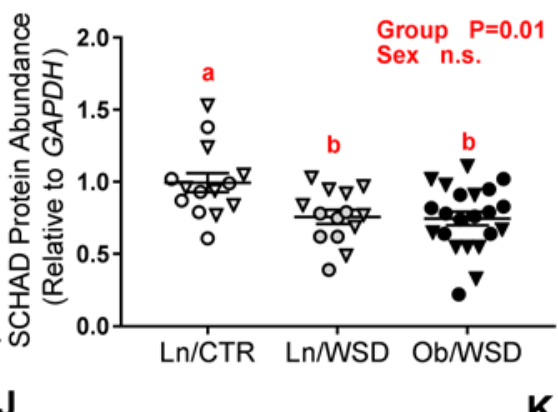

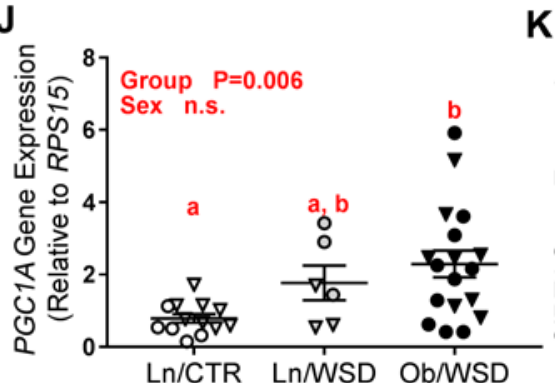

K

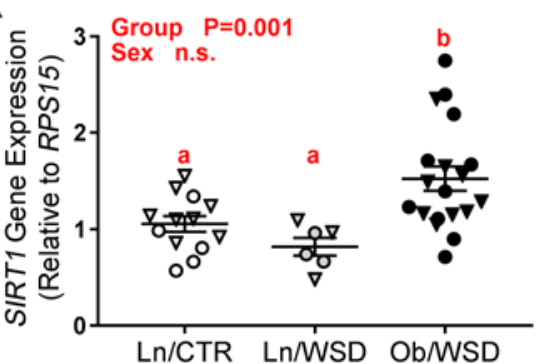

Figure 2. Maternal obesity combined with a Western-style diet (WSD) upregulates fatty acid metabolism in fetal muscle. Cellular lipids including (A) triglycerides, (B) fatty acids, and (C) phospholipids were extracted from fetal gastrocnemius (gastroc) and analyzed by mass spectrometry from male ( $M$, circles) and female (F, triangles) offspring of lean/overweight dams that had only received control diet (Ln/CTR; $n=5-6 \mathrm{M}, 6 \mathrm{~F}), \mathrm{Ln} / \mathrm{WSD}$ ( $n=5 \mathrm{M}, 5-6 \mathrm{~F}$ ), and obese dams chronically fed a WSD (Ob/WSD; $n=6-12$ M, 6-10 F) groups. Protein abundance for (D) CPT-1 $\beta$, (C) VLCAD, (H) SCHAD, and (I) LDH $\alpha$ was measured by Western blot in fetal muscle from Ln/CTR ( $n=5-7$ M, 7 F), Ln/WSD ( $n=6$ M, 6-8 F), Ob/WSD $(n=11$ M, 10-12 F). (F) Representative Western blots are shown for fatty acid transport and oxidation enzymes in male offspring. VLCAD was run in a separate gel from the other proteins listed. Data were adjusted to GAPDH. Gene expression of (E) PDK4, (J) PGC1A, and (K) SIRT1 was measured by qPCR and expressed relative to ribosomal protein S15 (RPS15) in fetal gastroc from offspring of Ln/CTR ( $n=5-6 \mathrm{M}, 8 \mathrm{~F}), \operatorname{Ln} / \mathrm{WSD}(n=3 \mathrm{M}, 3 \mathrm{~F}), \mathrm{Ob} / \mathrm{WSD}(n=10 \mathrm{M}, 8 \mathrm{~F})$. All data were analyzed by 2-way ANOVA (maternal group $\times$ fetal sex) with post-hoc tests. $P$ values for significant main effects are listed in each graph. Groups with the same letter are not significantly different from each other in post-hoc analysis. Individual data points and the group mean \pm SEM are shown.

rate-limiting enzyme in long-chain FA oxidation (FAO), was 2-fold higher in Ob/WSD compared with both Ln groups, independent of maternal diet (Figure 2, D and F). Similarly, gene expression of pyruvate dehydrogenase kinase 4 (PDK4) was increased in Ob/WSD compared with Ln/CTR (Figure 2E). The abundance of very-long-chain acyl-CoA dehydrogenase (VLCAD), specific for oxidation of long-chain FAs such as palmitoyl-CoA, myristoyl-CoA, and stearoyl-CoA, was $\sim 50 \%$ higher (Figure $2, \mathrm{G}$ and F), while short-chain 3-hydroxyacyl-CoA dehydrogenase (SCHAD) abundance was $~ 25 \%$ lower (Figure 2, H 
A

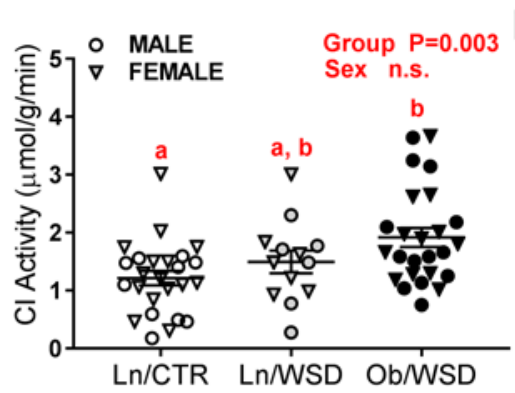

D

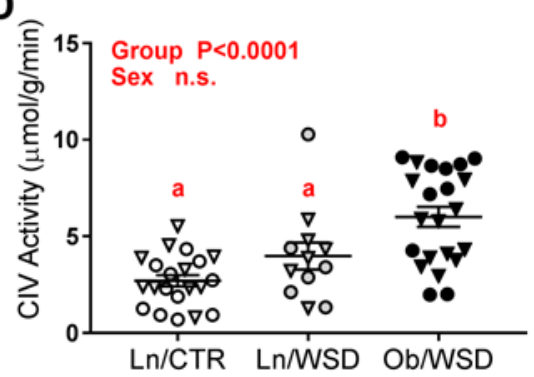

B

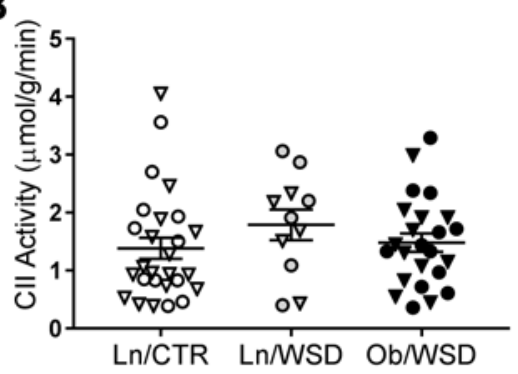

E

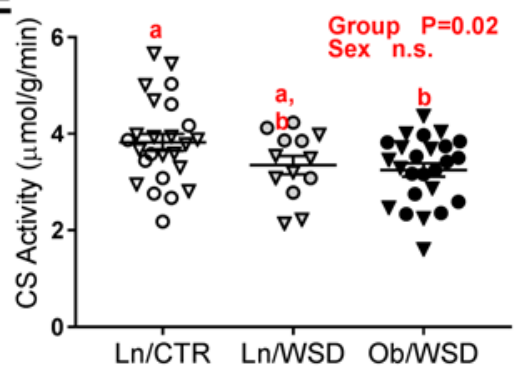

C

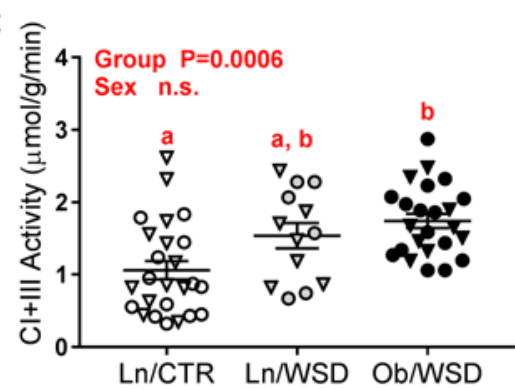

F

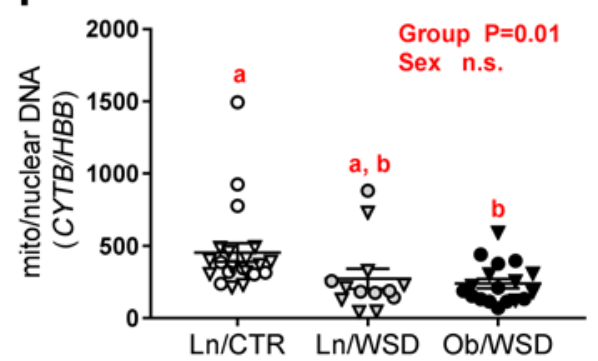

Figure 3. Maternal obesity combined with a Western-style diet (WSD) increases fetal muscle electron transfer system (ETS) activity but not mitochondrial abundance. ETS enzymatic activities for mitochondrial (A) complex (C) I, (B) CII, (C) Cl+III, and (D) CIV as well as (E) citrate synthase (CS) activity were measured by spectrophotometry in male (M; circles) and female ( $F$; triangles) fetal gastrocnemius muscle from lean/overweight dams that had only received control diet (Ln/CTR; $n=10-11 \mathrm{M}, 15-16 \mathrm{~F})$, Ln/WSD ( $n=6 \mathrm{M}, 5-7 \mathrm{~F})$, and obese dams chronically fed a WSD (Ob/WSD; $n=13 \mathrm{M}, 12 \mathrm{~F})$ groups. (F) An estimate of mitochondrial number was calculated as the ratio of expression for the mitochondrial gene, cytochrome $B(C Y T B)$, to the nuclear gene, hemoglobin beta (HBB), in fetal muscle from Ln/CTR $(n=9 \mathrm{M}, 12 \mathrm{~F}), \operatorname{Ln} / \mathrm{WSD}(n=8 \mathrm{M}, 5 \mathrm{~F})$ and Ob/WSD $(n=11 \mathrm{M}, 6 \mathrm{~F})$. Values are expressed relative to ribosomal protein $\mathrm{S} 15$ (RPS15). All data were analyzed by 2-way ANOVA (maternal group $\times$ fetal sex) with post-hoc tests. $P$ values for significant main effects are listed in each graph. Letters are used to indicate significant post-hoc differences. Groups with the same letter are not significantly different from each other. Individual data points and the group mean \pm SEM are shown.

and F) with WSD in both lean and obese groups. There was no difference in the abundance of long-chain acyl-CoA dehydrogenase (LCAD) or medium-chain acyl-CoA dehydrogenase (MCAD) (Supplemental Figure 2, A and B). Examining 2 enzymes that regulate the pool of cellular pyruvate, we found that lactate dehydrogenase $\alpha(\mathrm{LDH} \alpha)$ protein abundance was $25 \%$ lower (Figure 2, I and F) and glutamic-pyruvic transaminase 2 (GPT2) gene expression was 50\% higher (Supplemental Figure 2C) in Ob/WSD versus $\mathrm{Ln} / \mathrm{CTR}$. Expression of peroxisome proliferator-activated receptor $\gamma$, coactivator $1 \alpha(P G C 1 A)$ and sirtuin 1 (SIRT1), key transcriptional regulators of cellular metabolism, increased 2.5-fold and 1.5-fold (Figure 2, $\mathrm{J}$ and $\mathrm{K}$ ), respectively, with $\mathrm{Ob} / \mathrm{WSD}$. In contrast, there was no difference in mRNA expression of mitochondrial transcription factor A (TFAM) or mitofusin-2 (MFN2), key regulators of mitochondrial gene transcription and maintenance of the mitochondrial network, respectively (Supplemental Figure 2, D and E). Together, these data suggest that maternal obesity combined with WSD downregulates fetal muscle glucose utilization and upregulates the capacity for FAO.

Maternal obesity with WSD increases fetal ETS complex activity but not mitochondrial abundance in muscle. Complex I (CI) activity was 2-fold higher in Ob/WSD compared with Ln/CTR (Figure 3A). In contrast, $\mathrm{CII}$ activity was not different, suggesting a specific effect of maternal obesity on CI-driven oxidative phosphorylation (OXPHOS) in fetal muscle (Figure 3B). As electrons from $\beta$-oxidation can also enter at the Q-junction, we measured both NADH-cytochrome $c$ oxidoreductase (CI+III) activity as an indirect assessment of coenzyme Q sufficiency. CI+III activity was $75 \%$ higher in Ob/WSD compared with Ln/CTR, indicating no deficiency in coenzyme $\mathrm{Q}$ and likely reflecting the increase in $\mathrm{CI}$ activity (Figure 3C). CIII activity was not different between groups (Supplemental Figure 3A). CIV activity, the rate-limiting step in the ETS, was 3-fold higher in Ob/WSD compared with Ln/CTR (Figure 3D). Gene expression of the ATP synthase subunits ATP5C1 and ATP5A1 was $~ 2$-fold higher in Ob/WSD compared with Ln/CTR (Supplemental Figure 3, B and C). Despite higher ETS activity in Ob/WSD, citrate synthase (CS) activity, a marker of mitochondrial abundance, was 15\% lower compared with Ln/CTR (Figure 3E). In addition, the mitochondrial-to-nuclear DNA ratio was 2-fold lower in Ob/WSD versus Ln/CTR (Figure 3F). In contrast with our hypothesis, CI and CIV activity was unexpectedly higher with maternal obesity despite lower 
A

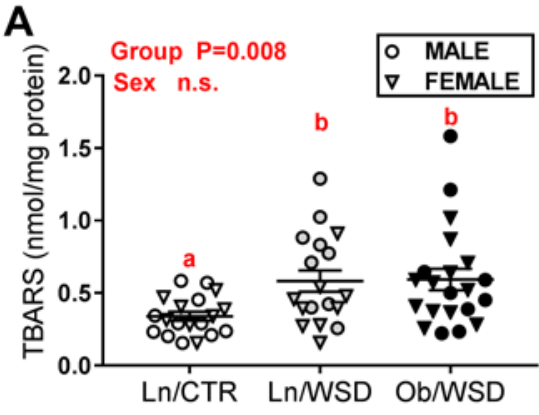

D

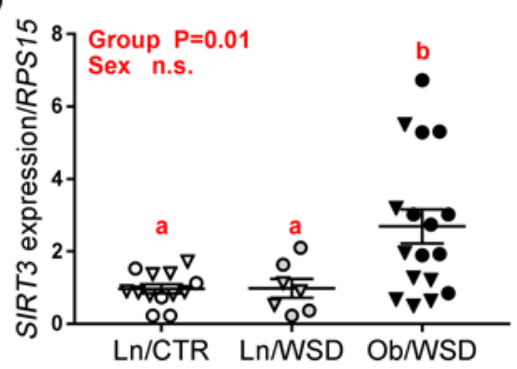

B

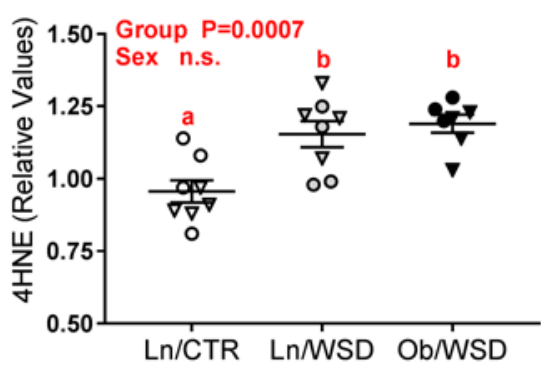

E

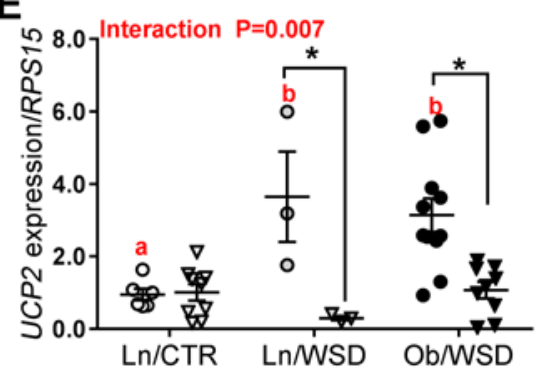

C

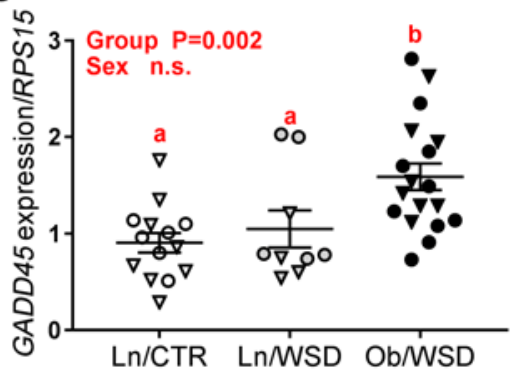

F

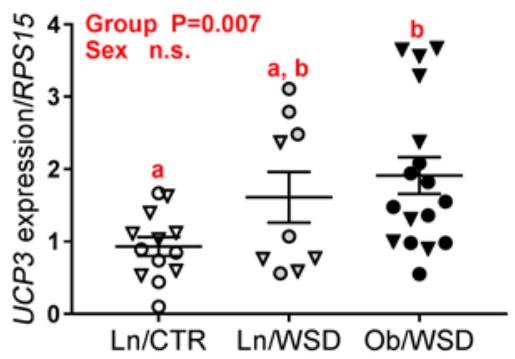

Figure 4. Maternal obesity combined with a Western-style diet (WSD) increases oxidative damage and mitochondrial uncoupling proteins in fetal muscle. (A) Lipid peroxidation was measured indirectly by thiobarbituric acid reactive substance (TBARS) assay in fetal gastrocnemius from male (M) and female (F) offspring of lean/overweight dams that had only received control diet ( $\mathrm{Ln} / \mathrm{CTR} ; n=11 \mathrm{M}, 8 \mathrm{~F}), \mathrm{Ln} / \mathrm{WSD}(n=9 \mathrm{M}, 8 \mathrm{~F})$, and obese dams chronically fed a WSD (Ob/WSD; $n=10 \mathrm{M}, 12 \mathrm{~F}$ ). (B) 4-hydroxynonenal-modified (4HNE-modified) proteins were measured by immunoblot ( $n=8 / \mathrm{group}$ ). Gene expression of (C) GADD45, (D) SIRT3, (E) UCP2, and (F) UCP3 was measured by qPCR and expressed relative to ribosomal protein S15 (RPS15) from Ln/CTR $(n=5-6 \mathrm{M}, 7-9 \mathrm{~F})$, Ln/WSD ( $n=3-5 \mathrm{M}, 3-4 \mathrm{~F})$ and Ob/WSD (19-11 M, 7-9 F). All data were analyzed by 2-way ANOVA (maternal group $\times$ fetal sex) with Tukey post-hoc tests. $P$ values for significant main effects are listed in each graph. Letters are used to indicate significant post-hoc differences $(P<0.05)$ between maternal groups. Bars with the same letter are not significantly different from each other. Asterisk $\left(^{*}\right)$ indicates significant difference between male and female offspring. Individual data points and the group mean \pm SEM are shown.

mitochondrial content, suggesting a compensatory upregulation in ETS enzymatic activity.

Oxidative stress is increased in fetal muscle exposed to maternal WSD. Considering that ETS activity was increased in Ob/WSD, we examined markers of oxidative stress in fetal muscle. Known markers of lipid peroxidation, thiobarbituric acid reactive substance (TBARS) and 4-hydroxynonenol-modified (4HNEmodified) proteins, were $\sim 2$-fold higher in fetal muscle exposed to a maternal WSD compared with CTR diet, independent of obesity (Figure 4, A and B). While there was no increase in total (combined mitochondrial and nuclear) DNA oxidation across groups (data not shown), there was a significant increase in the expression of the gene encoding growth arrest and DNA damage-45 (GADD45), a marker of cellular stress, in fetal muscle from obese versus lean dams (Figure 4C). Expression of SIRT3, a mitochondrial sirtuin that is thought to be essential in the induction of antioxidant system (37), was also 2.5-fold higher in Ob/WSD (Figure 4D). Upregulation of uncoupling protein 2 (UCP2) and UCP3 in skeletal muscle has been linked to an increase in FAO in rodent models $(38,39)$. In male offspring, UCP2 expression was 3 -fold higher in the maternal WSD groups compared with CTR, but was unchanged in female muscle (Figure 4E). In contrast, UCP3 expression was higher in male, and to a greater extent in female, fetal muscle from Ob/WSD, as compared with Ln/CTR. Together, these data suggest that maternal WSD, both alone and combined with obesity, increases ROS production and activates pathways to protect against cellular stress in fetal muscle.

Maternal obesity independently of diet reduces glucose oxidation in fetal muscle. To specifically test the impact of maternal obesity in the absence of WSD on functional changes in substrate utilization and oxidative capacity in offspring muscle, a cohort of obese adult females that were fed a WSD for $\sim 9$ years were switched to the healthy CTR diet prior to pregnancy (Ob/CTR). Measurements were made in the Ob/CTR group after switching to the healthy diet in the immediate prepregnancy interval. Prior to pregnancy, body weight was not different between $\mathrm{Ob} / \mathrm{WSD}$ and $\mathrm{Ob} / \mathrm{CTR}$ females but was significantly higher compared with $\mathrm{Ln} / \mathrm{CTR}$ females; however, by mid-third trimester, diet reversal led to a significant decrease in weight in Ob/CTR females, while other groups tended to gain weight (Supplemental Figure 4A). Similarly, percentage body fat was greater in $\mathrm{Ob} / \mathrm{WSD}$ and $\mathrm{Ob} / \mathrm{CTR}(41 \% \pm 2 \%$ and $37 \% \pm 3 \%$, respectively) compared 
A

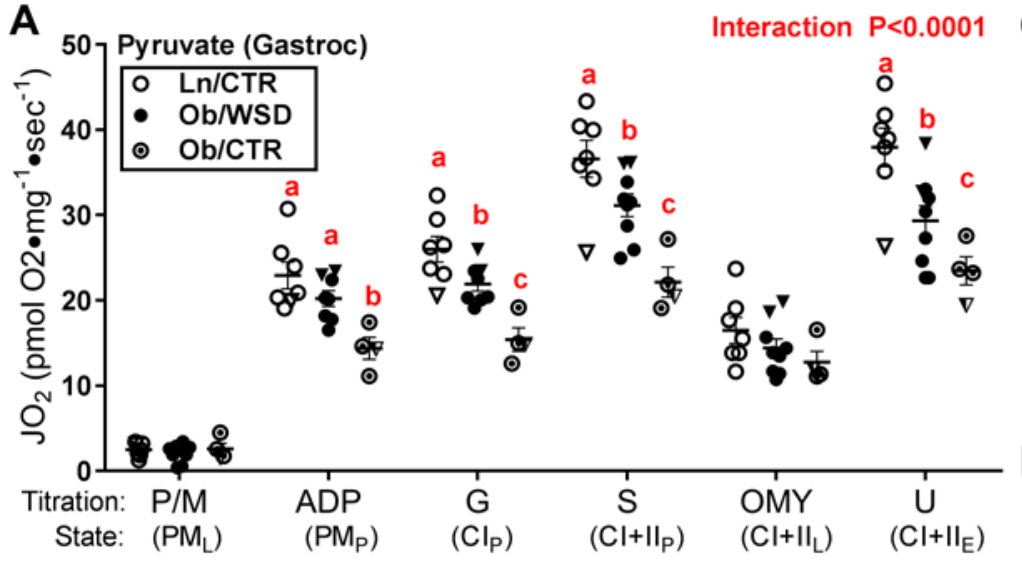

\section{B}

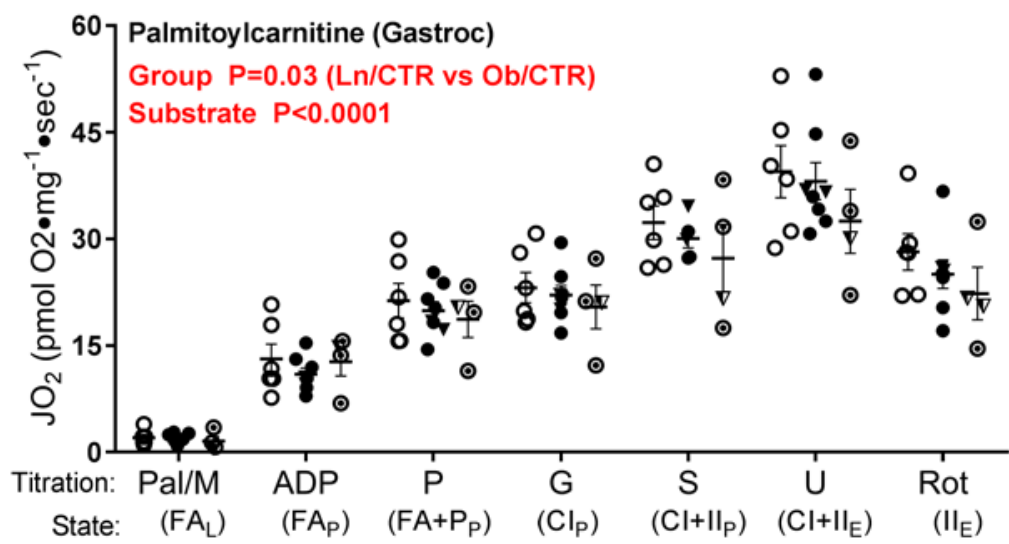

C

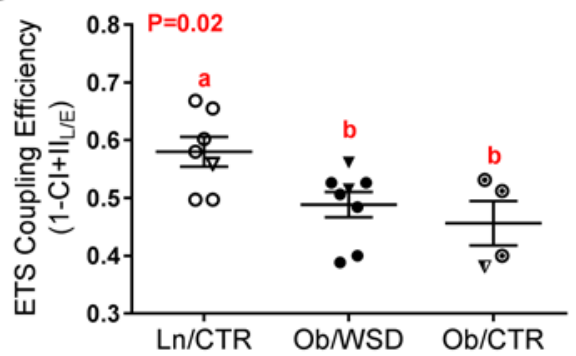

D

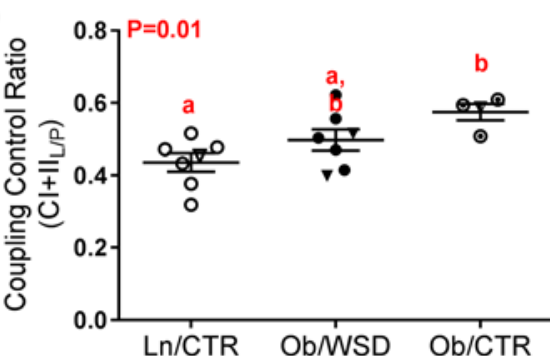

$E$

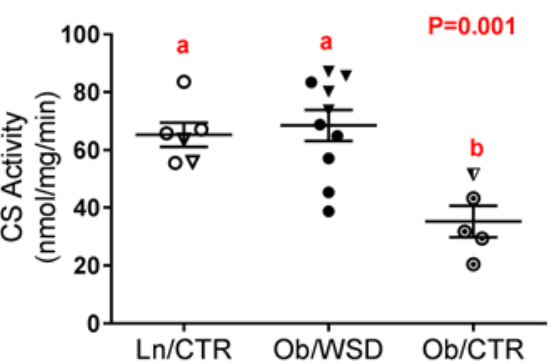

Figure 5. Reverting obese females to a healthy diet prior to pregnancy does not restore oxidative capacity in fetal gastrocnemius. (A) Respiratory flux $\left(\mathrm{JO}_{2}\right)$ adjusted according to muscle weight was measured after sequential addition of pyruvate/malate (P/M), ADP, glutamate (C), succinate (S), oligomycin (OMY) and the uncoupling agent carbonylcyanide $p$-trifluoromethoxy-phenylhydrazone (U) in permeabilized muscle fiber bundles (PMFBs) from male (circles) and female (triangles) fetal offspring of lean/overweight dams $(n=7)$ that had only received control diet (Ln/CTR), obese dams chronically fed a Western-style diet (Ob/WSD, $n=9$ ), and obese dams chronically ( $\leq 9$ years) fed a WSD before pregnancy but reverted to CTR diet prior to pregnancy ( $\mathrm{Ob} / \mathrm{CTR}, n=5)$. (B) JO , was measured after sequential addition of palmitoylcarnitine and malate (FA), ADP, pyruvate (FA+P), $G, S, U$, and the complex I inhibitor rotenone (Rot) in PMFBs from Ln/CTR $(n=5)$, Ob/WSD $(n=8)$ and Ob/CTR $(n=4)$. (C) Electron transfer system (ETS) coupling efficiency was calculated as $1-\left(\mathrm{Cl}+\mathrm{II}_{\perp}\right)$ divided by the subsequent noncoupled ETS capacity during the pyruvate protocol. (D) Coupling control ratio was calculated as leak respiration in the presence of $\mathrm{OMY}\left(\mathrm{Cl}+\mathrm{II}_{L}\right)$ divided by the preceding uninhibited oxidative phosphorylation flux $\left(\mathrm{Cl}+\mathrm{II}_{\mathrm{p}}\right)$ during the pyruvate protocol. $(\mathrm{E})$ Citrate synthase (CS) activity was measured in frozen tissues corresponding to the respirometry samples. Data were analyzed by 2-way ANOVA (maternal group $\times$ substrate) with Tukey post-hoc in $\mathbf{A}$ and $\mathbf{B}$ or 1-way ANOVA in $\mathbf{C}-\mathbf{E}$. Letters are used to indicate significant post-hoc differences $(P<0.05)$ between maternal groups. Groups with the same letter are not significantly different from each other. Individual data points and the group mean \pm SEM are shown.

with Ln/CTR females ( $25 \% \pm 2 \%$; Supplemental Figure 4B). In nonpregnant females, fasting insulin was higher with increased body fat in Ob/WSD and Ob/CTR (Supplemental Figure 4C). Prepregnancy insulin and glucose AUC during an i.v. GTT were not significantly different between groups. Insulin response during the third trimester i.v. GTT was significantly 2-fold lower in Ln/CTR and Ob/DR compared with $\mathrm{Ob} /$ WSD dams (Supplemental Figure 4E). Glucose AUC was not different between groups at either time point (Supplemental Figure 4, D and E).

For this experimental group, fetal skeletal muscle from Ob/CTR dams was compared with muscle from fetuses collected in that same year from obese females that remained on the WSD (Ob/WSD, $n$ $=10$ ) and lean or overweight females that were only fed CTR $\operatorname{diet}(\operatorname{Ln} / \mathrm{CTR}, n=7$; Ob/CTR, $n=5)$. Substrate utilization, mitochondrial efficiency, and respiratory capacity were measured in permeabilized muscle fiber bundles (PMFBs) from fetal gastrocnemius and soleus muscles. In response to pyruvate, PMFBs from $\mathrm{Ob} / \mathrm{WSD}$ compared with Ln/CTR had significantly reduced mitochondrial CI-dependent $\left(\mathrm{CI}_{\mathrm{P}}\right)$ and $\mathrm{CI}+\mathrm{II}$ respiratory capacity $\left(\mathrm{CI}+\mathrm{II}_{\mathrm{P}}\right)$ and uncoupled ETS capacity $(\mathrm{U})$ in gastrocnemius muscle (Figure 5A). Consistent with the aforementioned data demonstrating increased UCP content (Figure 4 , 
Table 1. Maternal diet and obesity alter accumulation of short-chain acylcarnitines in fetal muscle. Acylcarnitines were measured by UHPLC-MS in gastrocnemius from fetal offspring in each maternal group and normalized to a suitable stable isotope with either identical or similar chain lengths. Data are the mean \pm SEM.

\begin{tabular}{|c|c|c|c|c|c|c|c|}
\hline $\begin{array}{l}\text { Compound } \\
\text { (Chain Length) }\end{array}$ & std & Ln/CTR $(n=13-14)$ & Ln/WSD ( $n=11)$ & Ob/CTR $(n=6)$ & Ob/WSD $(n=13-14)$ & Diet & Obesity \\
\hline acyl-c2 & C2 & $12.517 \pm 1.26$ & $13.952 \pm 2.13$ & $12.632 \pm 1.40$ & $16.286 \pm 2.16$ & ns & ns \\
\hline acyl-C3 & C3 & $1.866 \pm 0.2$ & $1.880 \pm 0.35$ & $4.366 \pm 1.37^{A}$ & $2.704 \pm 0.57$ & ns & 0.009 \\
\hline acyl-C4 & C4 & $4.463 \pm 0.40$ & $3.940 \pm 0.98$ & $6.757 \pm 1.54$ & $5.143 \pm 0.58$ & ns & 0.02 \\
\hline acyl-C4-OH & ᄃ4 & $0.256 \pm 0.04$ & $0.237 \pm 0.04$ & $0.209 \pm 0.06$ & $0.291 \pm 0.06$ & ns & ns \\
\hline acyl-C4-DC & ᄃ4 & $0.334 \pm 0.03$ & $0.377 \pm 0.04$ & $0.443 \pm 0.03$ & $0.384 \pm 0.026$ & ns & ns (0.09) \\
\hline acyl-C5-OH & C5 & $0.788 \pm 0.09$ & $0.445 \pm 0.06^{A}$ & $0.856 \pm 0.04$ & $0.518 \pm 0.04^{\mathrm{B}}$ & $<0.0001$ & ns \\
\hline acyl-C6 & C5 & $0.075 \pm 0.009$ & $0.057 \pm 0.003$ & $0.064 \pm 0.006$ & $0.080 \pm 0.013$ & ns & ns \\
\hline acyl-c8 & ᄃ8 & $0.023 \pm 0.003$ & $0.021 \pm 0.003$ & $0.023 \pm 0.003$ & $0.030 \pm 0.004$ & ns & ns \\
\hline acyl-C8:1 & ᄃ8 & $0.003 \pm 0.0005$ & $0.002 \pm 0.0003$ & $0.003 \pm 0.0005$ & $0.004 \pm 0.0007$ & ns & ns \\
\hline acyl-C10 & ᄃ8 & $0.015 \pm 0.002$ & $0.015 \pm 0.002$ & $0.015 \pm 0.002$ & $0.020 \pm 0.002$ & ns & ns \\
\hline acyl-C14:1 & C14 & $0.093 \pm 0.021$ & $0.094 \pm 0.020$ & $0.111 \pm 0.039$ & $0.119 \pm 0.021$ & ns & ns \\
\hline acyl-C16 & C16 & $0.223 \pm 0.034$ & $0.188 \pm 0.044$ & $0.216 \pm 0.086$ & $0.204 \pm 0.015$ & ns & ns \\
\hline acyl-C16:1 & C16 & $0.093 \pm 0.018$ & $0.089 \pm 0.020$ & $0.093 \pm 0.034$ & $0.106 \pm 0.013$ & ns & ns \\
\hline acyl-C18:1 & C16 & $0.191 \pm 0.055$ & $0.195 \pm 0.062$ & $0.187 \pm 0.09$ & $0.189 \pm 0.014$ & ns & ns \\
\hline acyl-C18:2 & C16 & $0.149 \pm 0.036$ & $0.086 \pm 0.026$ & $0.115 \pm 0.056$ & $0.094 \pm 0.011$ & ns & ns \\
\hline acyl-C20:4 & C16 & $0.052 \pm 0.015$ & $0.040 \pm 0.015$ & $0.049 \pm 0.025$ & $0.031 \pm 0.003$ & ns & ns \\
\hline
\end{tabular}

Data were analyzed by 2-way ANOVA for maternal diet and obesity. $P$ values are listed for significant main effects. ${ }^{A}$ Significant difference vs. Ln/CTR. ${ }^{B}$ Significant difference vs. Ob/CTR. ns, no significant difference.

E and F) with maternal obesity, ETS coupling efficiency was also significantly lower in Ob/WSD (Figure $5 \mathrm{C}$ ). Calculation of the coupling control ratio revealed that the OXPHOS system exerted a greater limitation on the ETS in offspring from Ob/WSD compared with Ln/CTR (Figure 5D), corroborating findings of higher ETS enzymatic activity with maternal obesity (Figure 3, A and D). The decrease in oxidative metabolism with pyruvate was partially restored by prior addition of FA (palmitoylcarnitine) substrate along with pyruvate (Figure $5 \mathrm{~B}$ ) to PMFBs from Ob/WSD versus Ln/CTR; however, there was still an overall decrease in fetal oxidative metabolism with maternal obesity $(P=0.03)$. Surprisingly, the decrease in oxidative metabolism and mitochondrial efficiency was worsened for both pyruvate and palmitoylcarnitine protocols in PMFBs from Ob/CTR compared with Ob/WSD or Ln/CTR offspring (Figure 5, A and B), suggesting that switching obese mothers to a healthy diet alone may not be sufficient for improved metabolic outcomes in the offspring. CS activity, although not different between Ln/ $\mathrm{CTR}$ and $\mathrm{Ob} / \mathrm{WSD}$ in this cohort, was significantly reduced in the Ob/CTR and may partially explain the further reduction in oxidative metabolism (Figure 5E). The respiratory flux data (Figure 5, A and B) were not corrected for mitochondrial content, but expressed relative to muscle weight. Together, these data indicate that offspring exposed to maternal obesity may have reduced glucose oxidation and an increased reliance on FA substrates. In parallel studies in PMFBs from the soleus, there was no difference in oxidative metabolism in fetal muscle across maternal groups, indicating that the above responses are fiber-type and/or muscle specific (Supplemental Figure 5). Additionally, these results suggest that maternal metabolic status, rather than dietary intake alone, can alter mitochondrial substrate utilization, reduce OXPHOS capacity, and decreases efficiency in fetal skeletal muscle.

To further examine substrate utilization, we quantified endogenous acylcarnitine levels relative to isotope-labeled standards from gastrocnemius muscle of fetal offspring from lean and obese dams consuming either CTR or WSD during pregnancy. Consistent with the respirometry data (Figure 5B), there was no 
A

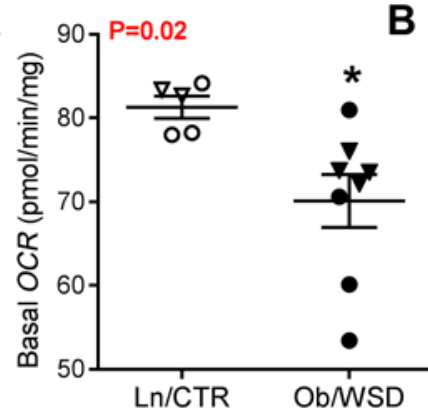

B

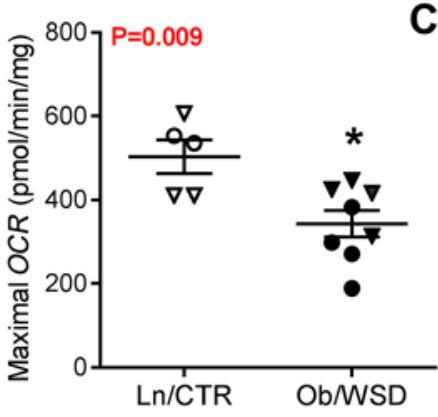

C

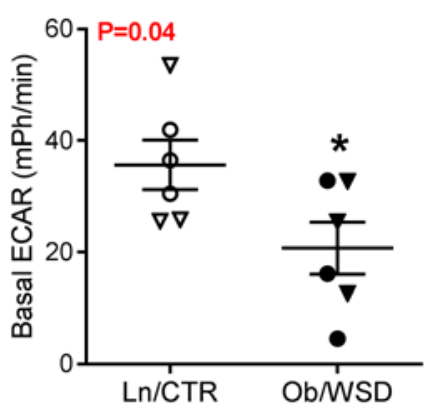

Figure 6. Exposure to maternal obesity leads to a persistent decrease in offspring muscle oxidative metabolism. Myoblasts were isolated from male (circle) and female (triangle) rectus femoris muscle from fetal offspring of lean dams on control diet (Ln/CTR) and obese dams on a Western-style diet (Ob/WSD). (A) Basal oxygen consumption rate (OCR) and (B) maximal OCR after addition of carbonylcyanide $p$-trifluoromethoxy-phenylhydrazone were measured in differentiated myotubes from $\operatorname{Ln} / \operatorname{CTR}(n=5)$ and Ob/WSD $(n=8)$. (C) Extracellular acidification rate (ECAR), an indirect marker of glycolysis, was also measured in differentiated myotubes from Ln/CTR $(n=6)$ and Ob/WSD $(n=6)$. Data were analyzed by unpaired Student's $t$ test and significant $P$ values are listed in each graph. Experiments were run in at least triplicate and values averaged for each sample. The averaged data from each sample and the group mean \pm SEM are shown.

difference in long (C12-C20) or medium chain (C6-C10) acylcarnitine accumulation with maternal diet or obesity in fetal muscle. This lack of an increase in long-chain acylcarnitines may be partially related to the increase in protein abundance of VLCAD (Figure 2G) and CPT-1ß (Figure 2D). In contrast, maternal obesity significantly increased the accumulation of short-chain acylcarnitines (C3, C4, C5, C5:1) in fetal muscle (Table 1). The increase in these short-chain acylcarnitines is consistent with an elevation in branched-chain amino acid (BCAA) catabolism. Surprisingly, acyl-C5 and -C5:1 were lower in muscles from WSD compared with CTR offspring in each phenotype (Table 1). This suggests that WSD is either leading to less BCAA mobilization or availability or increased efficiency of BCAA catabolism.

We also measured the relative metabolite concentration for other major energy pathways. We found a trend for overall lower glucose utilization through glycolysis and the TCA cycle in fetal muscle from both lean and obese dams on WSD (Supplemental Figure 6). Several of the intermediates showed significant differences by independent-samples Student's $t$ test compared with Ln/CTR, but a large intracohort variation prevented a clear statistical interpretation of the changes. Pathway enrichment analysis of the metabolomics data was performed using MetPa (40), illustrating the major pathways affected when comparing Ob/ WSD and Ln/CTR. Pathways that scored with the largest impact included glycolytic, TCA, and purine metabolic pathways ( $P$ values of $0.05,0.06$, and 0.06 , respectively, but $P>0.1$ after false discovery correction for multiple comparison testing). Further studies using larger cohorts may be required to validate the perturbations described for these pathways. Overall, these results support the findings that maternal obesity alters fetal muscle substrate utilization, resulting in reduced fetal muscle glucose utilization and an upregulation of FA metabolism.

Metabolic reprogramming in offspring muscle by maternal WSD in the presence of concomitant maternal obesity is persistent. To determine if the decrease in oxidative metabolism in fetal muscle is maintained after the influence of the maternal-fetal obesogenic environment is removed, we isolated primary myoblasts from fetal rectus femoris from Ln/CTR or Ob/WSD groups and cultured cells for 2 to 3 passages in normal growth media. Both basal and maximal respiration (Figure 6, A and B), and glycolytic flux (Figure 6C) were $\sim 30 \%-40 \%$ lower in differentiated myotubes from male and female Ob/WSD compared with Ln/ CTR. The reduced glycolytic flux in Ob/WSD myotubes matches the overall $40 \%$ decline in respiration seen in isolated fibers with pyruvate substrate (Figure 5A). Together, these data show that fetal exposure to maternal obesity combined with WSD leads to a persistent decrease in offspring glucose metabolism and oxidative capacity.

\section{Discussion}

In the current study, we used a well-established nonhuman primate model of maternal overfeeding to investigate the impact of maternal WSD alone, or combined with obesity, on metabolic programming in fetal 
skeletal muscle. Using this model, we have previously demonstrated that exposure to maternal WSD and obesity during pregnancy leads to steatosis and oxidative stress in fetal liver (35) and fetal pancreas (41), 2 organ systems involved in glucose homeostasis, as well as effects on fetal systems that regulate food intake (42) and energy balance $(43,44)$. We extend these findings, being to the best of our knowledge the first to report that maternal obesity leads to reduced mitochondrial content, oxidative capacity, and OXPHOS efficiency in fetal skeletal muscle. Furthermore, we find that fetal muscle adapts to the obesogenic fetal environment by upregulating FAO, as well as CPT-1 $\beta$ protein abundance and $P D K 4$ expression to promote lipid utilization over glucose oxidation. The decrease in maximal oxidative capacity and reduced glucose metabolism was persistent in isolated primary fetal myotubes from offspring of Ob/WSD compared with Ln/CTR dams, implying that maternal obesity, especially when combined with a WSD, creates an adverse fetal environment that results in reprogramming of skeletal muscle oxidative metabolism. These changes in oxidative metabolism may have important implications for future metabolic health.

Reduced muscle oxidative metabolism is linked to muscle insulin resistance, a key predictor for the development of metabolic diseases $(45,46)$. Considering that offspring of obese and/or diabetic mothers have a 2-fold higher risk of developing metabolic syndrome in childhood $(5,6)$, a key issue that we sought to address was the combined, and independent, effects of maternal WSD and obesity on fetal muscle substrate metabolism and mitochondrial function. We used 2 different models to address this question. First, we investigated metabolic pathways and ETS activity in fetal muscle of offspring of lean or obese dams chronically consuming the WSD compared with lean dams on a healthy diet. Fetal skeletal muscle from obese dams on the WSD had profound changes in key regulators of substrate metabolism (e.g., CPT- $1 \beta$, PDK4, PGC1A, or SIRT1), ETS enzymatic activity, as well as an increase in short-chain acylcarnitine concentration, indicative of elevated BCAA catabolism. Our analysis also revealed that maternal WSD alone suppressed protein levels of SCHAD and increased abundance of VLCAD, and increased measures of oxidative stress. Additionally, WSD partially suppressed the increase in short-chain acylcarnitine accumulation independently of maternal phenotype in fetal muscle. These data suggest that fetal skeletal muscle is highly susceptible to WSD, and when combined with maternal obesity overwhelms metabolic enzymes and ETS activity, resulting in substantially reduced oxidative capacity and elevated oxidative stress.

To further isolate the impact of maternal obesity from the effects of WSD on fetal muscle oxidative metabolism, we used a dietary intervention model in which obese females were switched to a CTR diet prior to pregnancy and compared them with obese dams that remained on a WSD. Based on previous studies using this model (35), we predicted that reverting mothers to a healthy diet would improve fetal muscle outcomes despite the obese phenotype. In contrast with our hypothesis, reverting obese dams to a CTR diet in the setting of maternal obesity did not improve mitochondrial oxidative capacity. In fact, fetal muscle oxidative capacity in the presence of pyruvate was further reduced $(P<0.05)$ in the Ob/CTR compared with $\mathrm{Ob} / \mathrm{WSD}$ muscle (Figure 5A); additionally, the $\mathrm{Ob} / \mathrm{CTR}$, unlike the $\mathrm{Ob} / \mathrm{WSD}$, showed a significant reduction in the presence of lipid (Figure $5 \mathrm{~B}$ ). This suggests that the maternal obese metabolic environment, even when on a healthy diet, can reduce oxidative capacity and decrease mitochondrial efficiency in fetal skeletal muscle. It should be noted that only the dams in the Ob/CTR group lost weight during pregnancy and that this may at least be partially responsible for the lack of an improvement in fetal muscle metabolism. A limitation of this model was that we were not able to also compare the effects of WSD in lean dams on fetal substrate metabolism. However, in a rat model, a maternal WSD also downregulated gene pathways related to OXPHOS, ETS enzymes, and ATP synthesis in the skeletal muscle from adult male offspring (29). While these data did not directly measure mitochondrial function, they add to our findings that skeletal muscle mitochondrial metabolism is very likely programmed in utero by exposure to WSD during gestation.

Mechanistically, we believe the decrease in respiratory capacity results from a combination of decreased ETS efficiency and decreased mitochondrial content and not a deficiency in ETS enzymatic activity per se. The higher ETS activity is likely a compensatory mechanism related to lower mitochondrial content, as we observed higher ETS enzymatic activity in fetal muscle from Ob/WSD dams. Lower mitochondrial content has also been reported in umbilical cord from large- and small-for-gestational-age (LGA and SGA, respectively) infants compared with appropriate-weight-for-gestational-age (AGA) infants (47), suggesting that a common mechanism in models of over- and undernutrition may influence mitochondrial accumulation and contribute to the increased metabolic disease risk found in both LGA and SGA infants. The decrease in markers of mitochondrial content occurred despite an upregulation of PGC1A expression, an important coactivator in mitochondrial biogenesis, indicating 
a potential disconnect in cell signaling; however, PGC-1 $\alpha$ protein localization and acetylation status, markers of coactivator function, were not measured. Two other mitochondrial transcriptional regulators, TFAM and MFN2, were not increased, which suggests a lack of a coordinated induction of mitochondrial biogenesis in fetal muscle in response to maternal obesity or WSD. Importantly, the fetal phenotype of impaired oxidative metabolism was persistent in primary fetal myotubes, indicating that these changes are not just an adaptation to the current fetal environment but a persistent alteration in the metabolic phenotype of the muscle.

In fetal muscle from obese dams, the decrease in oxidative capacity was more pronounced in response to nonlipid substrates (pyruvate) and was partially relieved with addition of palmitoylcarnitine, despite an adequate supply of both during the experimental condition in the permeabilized fibers. We propose that an obesogenic fetal environment promotes an increased reliance on lipid oxidation. The ability of pyruvate to contribute to TCA cycle intermediates and oxidative metabolism is dependent, in part, on PDK4, a key regulator in the metabolic drive towards FAO in skeletal muscle $(48,49)$. Increased PDK4 expression has previously been shown in fetal muscle from growth-restricted sheep and was inversely correlated with glucose-oxidation capacity in muscle (50). Along these lines, fetal muscle from obese compared with lean dams had a 2-fold increase in PDK4 expression as well as lower lactate dehydrogenase $\alpha$ abundance and higher GPT2 expression, suggesting a diversion of pyruvate away from oxidation. In insulin-sensitive adults, raising plasma FA concentration increases the capacity for FAO in muscle through a coordinate upregulation of FAO enzymes MCAD, VLCAD, and CPT-1 $\beta$, and ETS complex subunits (51-54). Moreover, upregulation of these FAO enzymes in skeletal muscle is regulated at the transcriptional level by PPAR $\delta$ and PGC1 $\alpha(37,51)$. We found a similar pattern of upregulation in PGC1A, and downstream targets, CPT-1 $\beta$ and VLCAD, as well as an increase in mitochondrial ETS activity, providing additional support for a metabolic shift towards increased FAO.

Oxidative stress and mitochondrial ROS generation are typically balanced by the cellular antioxidant defense system, and during development are upregulated only in the latter part of gestation (55). As such, early exposure to oxidative stress, as seen in preterm infants, is associated with greater ROS production and lower concentrations of antioxidant enzymes, which results in an increased susceptibility to oxidative damage (56). Maternal obesity is also associated with higher oxidative stress and reduced placental antioxidant status (57). In our model, WSD alone increased muscle lipid peroxidation, but maternal obesity was also required to increase SIRT3 expression, a key regulator in mitochondrial antioxidant defense $(58,59)$, and GADD45, a cellular stress sensor, indicating a graded increase in cellular oxidative stress in fetal muscle with a worsening maternal phenotype. In addition to antioxidant enzymes, UCPs, especially UCP3, are rapidly induced in response to FAs and ROS itself $(60,61)$ as a protective mechanism to reduce ROS production and subsequent oxidative damage (61-63). UCP2 and UCP3 have also been proposed to play a role in regulating lipid metabolism (38). For example, UCP2 has been shown to limit glucose oxidation by shuttling oxaloacetate out of the mitochondria (64). Interestingly, UCP3 expression was 2-fold higher in both male and female offspring muscle from offspring of obese mothers, again suggestive of increased oxidant stress, while UCP2 expression was nearly 4-fold higher in response to both maternal WSD and obesity, but only in male offspring. Sex differences in the offspring response to maternal diet or obesity, while not overt in our study, were associated primarily with lipid metabolism (e.g., free FAs, diacylglycerols, LCAD) and measures of oxidative stress. Female offspring had less induction of stress pathways compared with male offspring, with the greatest variability seen in response to maternal diet alone (e.g., Ln/WSD). Barker and Thornburg (65) have proposed that differences in placental adaptation to an adverse maternal-fetal environment may underlie the different susceptibility to metabolic diseases, which may also explain differences in metabolic programming in male and female offspring (66).

Identifying interventions for an obese pregnancy that may prevent, or ameliorate, the intergenerational effects of maternal obesity is of high clinical relevance. For example, we have previously shown that reverting mothers to a healthy diet at the start of pregnancy improved but did not normalize the fetal liver phenotype (35) and prevented WSD-induced changes in epigenetic patterning (67). Herein, however, diet reversal in obese dams failed to significantly restore metabolic outcomes in fetal muscle. However, these studies are limited to the fetal interval, and it is not yet known if these or similar observations will hold true in the postnatal interval. It is notable that, unlike the fetal skeletal muscle, the liver $(35,68)$ as well as the pancreas $(41,69)$ are equally sensitive to both maternal WSD and maternal obesity; that is, maternal WSD, independent of maternal obesity, was sufficient to drive the metabolic reprogramming and alter cellular development in these fetal tissues. 
In summary, these findings provide further evidence for complicated, substantial, and tissue-specific physiological interactions between maternal-diet phenotypes and skeletal muscle mitochondrial energetics. Our results reveal that there are global changes in lipid and mitochondrial metabolic pathways in the fetus that are predictive of later-onset metabolic disease and obesity. A major unanswered question is how fuel overload and/or other fetal insults mechanistically program mitochondrial dysfunction and reduce the ability to oxidize fuels in the postnatal period. While correlations exist between excess maternal lipid exposure, mitochondrial dysfunction, and obesity in the offspring, there are no data in human infants, nor are the early mechanisms for programming mitochondria, perhaps through epigenetic pathways, preceding obesity fully understood. Thus, further research is necessary, as well as controlled, well-designed interventions in mothers to mitigate this risk in the next generation. While the current studies are limited to the early third trimester fetal muscle, future efforts will be aimed at examining neonatal and juvenile offspring.

\section{Methods}

Experimental design and model. This nonhuman primate model has been previously described (35). Briefly, adult Japanese macaques were socially housed in indoor/outdoor pens and were maintained on a CTR $\operatorname{diet}(15 \%$ calories from fat) or WSD (37\% calories from fat) for 2 to 7 years prior to pregnancy. Measurement of body weight, fasting plasma for assay of glucose, TAG, and insulin concentrations were collected prior to an i.v. GTT in the nonpregnant state and during the third trimester of pregnancy as previously described (35). The AUC for glucose and insulin was calculated from baseline insulin and glucose values. For this study, dams were classified as lean or obese based on percentage body fat obtained by duel-energy X-ray absorptiometry (DEXA). The baseline population used to define maternal obesity consisted of 33 age-matched adult females consuming the CTR diet. Percentage body fat was $16.5 \% \pm 7.5 \%$ (mean \pm SD). For females on the WSD, lean was defined as body fat less than $25 \%$ and obese as greater than $30 \%(\sim 2$ $\mathrm{SD}$ above baseline mean). These groupings are consistent with validation of body condition scoring using DEXA in rhesus macaques such that animals with an optimal body conditioning score (BCS) of 3.0 had on average $25 \%$ body fat, while animals with BCS of 4.0 and 4.5 (heavy to obese) had $35 \%-40 \%$ body fat (70). Females were allowed to breed seasonally, and gestational age was determined by ultrasound (36). Pregnancies were terminated by cesarean section at gestational day 130 .

Maternal dietary intervention. A cohort of obese females that had been on the WSD for $\sim 9$ years were switched to CTR diet prior to pregnancy ( $65 \pm 24$ days) and were maintained on the CTR diet for the duration of the pregnancy (Ob/CTR). Females that were continuously maintained on WSD or CTR diet with pregnancies in that same year were used as controls for the intervention group. Maternal measurements for body weight, and plasma glucose and insulin in the fasting state and during i.v. GTT, were taken before the dietary reversal intervention in the nonpregnant state and during the intervention period in the third trimester of pregnancy (Supplemental Figure 4). For the intervention year, a total of $7 \mathrm{Ln} / \mathrm{CTR}, 8 \mathrm{Ob} / \mathrm{WSD}$, and $5 \mathrm{Ob} / \mathrm{CTR}$ fetal offspring were studied.

Fetal muscle collection. At gestational day 130, fetuses were delivered by cesarean section and necropsied. Fetal skeletal muscle including gastrocnemius (gastroc), soleus, vastus lateralis, and rectus femoris were rapidly dissected of fascia and portions were flash frozen in liquid nitrogen-cooled isopentane, transferred to a biopsy preservation solution for respiration studies, or placed into sterile F10 media for isolation of primary fetal myoblasts. Frozen tissue was stored at $-80^{\circ} \mathrm{C}$ until analysis. A total of $25 \mathrm{Ln} / \mathrm{CTR}$ (12 male [M], 13 female [F]), 18 Ln/WSD (10 M, 8 F), 32 Ob/WSD (20 M, 12 F) fetal offspring were studied; however, not all samples were analyzed in every assay.

Immunoblot assays. Frozen gastroc (30 mg) was homogenized as previously described (35). Protein concentration was determined using the BCA Protein Assay kit (Pierce). For immunoblot assay, $50 \mu \mathrm{g}$ protein homogenate was resolved in a 7\% acrylamide Tris-acetate gel (Life Technologies) and transferred to a polyvinylidene fluoride membrane (Bio-Rad Laboratories). Membranes were probed with appropriate primary and secondary antibodies. Immunoreactive products were detected using Western Lightning enhanced chemiluminescence (PerkinElmer) and exposure to X-OMAT sheet film (Eastman Kodak Co.). Images were scanned and bands quantified using ImageJ software (NIH) and expressed relative to an internal standard run in each gel. Glyceraldehyde-3-phosphate dehydrogenase (GAPDH) was measured as a loading control. Quantification of $4 \mathrm{HNE}$-modified proteins was performed on all protein bands from 170 to $25 \mathrm{kDa}$ and reported relative to control in each sex. Primary antibodies are listed in Supplemental Table 1. 
$R N A$ isolation and quantitative PCR. RNA was isolated from $50 \mathrm{mg}$ gastroc using the RNeasy Plus Mini Kit with on-column DNA digest (QIAGEN). RNA was quantified, reverse transcribed (ImPromII reverse transcriptase; Promega), and gene expression levels determined by quantitative real-time PCR on a Roche LightCycler 480 instrument. An internal standard was run on all plates. Data were adjusted to ribosomal protein S15 (RPS15). Primer sequences are listed in Supplemental Table 2.

Mitochondrial enzymatic activity assays. Frozen, powdered gastroc was weighed (30-50 mg) and homogenized on ice with glass-on-glass homogenizing tubes in $0.5 \mathrm{ml}$ ice-cold Zheng buffer ( $210 \mathrm{mM}$ mannitol, $70 \mathrm{mM}$ sucrose, $5 \mathrm{mM}$ HEPES, and $1 \mathrm{mM}$ EGTA, pH to 7.2 using potassium hydroxide). Sample homogenates were divided into aliquots and then underwent 3 freeze-thaw cycles using a methanol/dry ice bath for enzyme analysis of respiratory complexes (C) I+III, II, II+III, III and IV. Homogenates were further disrupted by sonication for enzyme analysis of CI and CS. Enzymatic activities were measured by spectrophotometric assays as previously described (71) with minor modification. All assays were performed in 96-well plates using a Molecular Devices SpectraMax 190 spectrophotometer at $30^{\circ} \mathrm{C}$ in a final volume of $0.25 \mathrm{ml}$ in $50 \mathrm{mM}$ potassium phosphate buffer (KPi, pH 7.4) unless otherwise indicated. For all assays, muscle homogenate was diluted to varying degrees with KPi buffer and reactions evaluated for up to 40 minutes to determine the optimal dilution needed to maximize the linearity of the reaction. Enzymatic activity was calculated according to the following equation using the appropriate extinction coefficient for

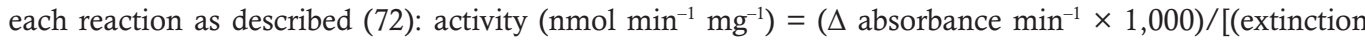
coefficient $\times$ volume of sample used in $\mathrm{ml}) \times\left(\right.$ sample protein concentration in $\left.\left.\mathrm{mg} \mathrm{ml}^{-1}\right)\right]$.

Lipid analysis. Fetal gastroc $(50 \mathrm{mg})$ was dissected of extramuscular adipose tissue and fascia, lyophilized, weighed, and homogenized in methanol along with an internal standard of tripentadecanoic acid (Omni International). Skeletal muscle lipid extraction, isolation of intramuscular TAGs, diacylglycerides, FAs, and phospholipids, and analysis by gas chromatography/mass spectrometry were performed as previously described (73).

Lipid peroxidation (TBARS). Lipid peroxidation was measured using a commercially available kit in which the reaction of malondialdehyde, a lipid peroxidation product, with TBARS is measured spectrophotometrically (Cayman Chemical). Briefly, $25 \mathrm{mg}$ of powdered gastroc was homogenized on ice in $0.5 \mathrm{ml}$ PBS using glass-on-glass homogenizers, then sonicated for 15 seconds and $100 \mu$ was heated to $95^{\circ} \mathrm{C}$ with TBARS for 1 hour. After heating, samples were centrifuged at $1,650 \mathrm{~g}$ for 15 minutes, supernatant and standards were added to a 96-well plate, and the absorbance read at $532 \mathrm{~nm}$. Data were normalized to protein concentration.

DNA isolation and amplification. For DNA extraction, $25 \mathrm{mg}$ gastroc was digested in $0.25 \mathrm{ml}$ buffer containing $100 \mathrm{mM} \mathrm{NaCl}, 10 \mathrm{mM}$ Tris, $\mathrm{pH} 8.0,25 \mathrm{mM}$ EDTA, and $0.25 \%$ SDS with $0.1 \mathrm{mg} / \mathrm{ml}$ proteinase $\mathrm{K}$ at $50^{\circ} \mathrm{C}$ overnight. An equal volume of phenol/chloroform/isoamyl alcohol (25:24:1) was added, mixed by shaking, and centrifuged for 10 minutes at $1700 \mathrm{~g}$ to separate the phases. The aqueous layer was transferred to a new tube with $0.025 \mathrm{ml} 3 \mathrm{M} \mathrm{Na}$ acetate and $0.5 \mathrm{ml}$ ice-cold $70 \%$ ethanol. Samples were cooled for 10 minutes on ice, centrifuged at $10,000 \mathrm{~g}$, and then the DNA pellet was resuspended in $0.1 \mathrm{ml}$ solution with $10 \mathrm{mM}$ Tris, $\mathrm{pH} 8.0$ and $0.1 \mathrm{mM}$ EDTA. DNA concentration and purity were determined using a NanoDrop 2000c spectrophotometer (Thermo Fisher). DNA was analyzed by quantitative real-time PCR for mitochondrial gene (cytochrome B) and nuclear gene ( $\beta$-globin) expression with a Roche LightCycler 480 instrument using a standard curve to determine the efficiency of each reaction.

PMFB preparation and respiration. Fetal gastroc and soleus muscles (10-20 mg), collected at necropsy, were immediately placed into $2 \mathrm{ml}$ ice-cold biopsy preservation solution (BIOPS), containing $10 \mathrm{mM}$ Ca-EGTA buffer, $0.1 \mu \mathrm{M}$ calcium, $20 \mathrm{mM}$ imidazole, $20 \mathrm{mM}$ taurine, $5 \mathrm{mM}$ K-MES, $0.5 \mathrm{mM}$ DTT, $6.56 \mathrm{mM} \mathrm{MgCl}_{2}, 5.77 \mathrm{mM}$ ATP, $15 \mathrm{mM}$ phosphocreatine, pH 7.1 and shipped overnight to Colorado State University or to the University of Oregon for mitochondrial respiratory analysis. Mitochondrial integrity was confirmed in all samples by examining respiratory responses to $10 \mu \mathrm{M}$ cytochrome $c$, which should be negligible in mitochondria with intact outer membranes. Any samples showing a greater than $10 \%$ increase in respiratory flux under near-maximal OXPHOS states were excluded from the study. Muscles were dissected in ice-cold BIOPS and teased fiber bundles (5-8 mg) were permeabilized in BIOPS with $30 \mu \mathrm{g} / \mathrm{ml}$ saponin for 20 minutes, washed, and weighed as described (74). Mitochondrial respiratory function was measured in PMFBs by high-resolution respirometry using an Oxygraph-2K system (Oroboros Instruments). All respirometry data were collected at $37^{\circ} \mathrm{C}$ in a hyperoxygenated environment (250-400 $\mu \mathrm{M} \mathrm{O}_{2}$ ) to avoid potential oxygen diffusion limitations in PMFBs (75). Two titration protocols were run in parallel to measure mitochondrial function as previously described (74). For 
protocol 1, sequential additions of $5 \mathrm{mM}$ pyruvate and $2 \mathrm{mM}$ malate, $4 \mathrm{mM}$ ADP, $10 \mathrm{mM}$ glutamate, $10 \mathrm{mM}$ succinate, $10 \mu \mathrm{M}$ cytochrome $c, 2 \mu \mathrm{g} / \mathrm{ml}$ oligomycin, followed by addition of the uncoupling agent carbonylcyanide $p$-trifluoromethoxy-phenylhydrazone (FCCP) in $0.5-\mu \mathrm{M}$ steps, to each chamber. For protocol 2, sequential additions of $40 \mu \mathrm{M}$ palmitoylcarnitine and $2 \mathrm{mM}$ malate, $4 \mathrm{mM}$ ADP, $5 \mathrm{mM}$ pyruvate, $10 \mathrm{mM}$ glutamate, $10 \mathrm{mM}$ succinate, $10 \mu \mathrm{M}$ cytochrome $c, 0.5-\mu \mathrm{M}$ steps of $\mathrm{FCCP}$, and then $0.5 \mu \mathrm{M}$ rotenone were added to a second chamber. These protocols provide a comprehensive evaluation of muscle respiratory capacity and substrate control during OXPHOS (P state), as well as the extent of nonphosphorylating respiratory leak (L state) and the noncoupled enzymatic capacity of the ETS (E state). Selected flux control ratios were calculated for determination of respiratory coupling, leak, and substrate control in each muscle type.

Myoblast isolation and respirometry. Myoblasts were isolated from rectus femoris muscle immediately following necropsy as previously described (76), with minor modifications. Muscle (0.5-2 g) was minced and digested in collagenase type 2, filtered (LS004204, Worthington Biochemical) at a concentration of $25 \mathrm{mg}$ collagenase $/ 2 \mathrm{~g}$ muscle $(\sim 90 \mathrm{U} / \mathrm{ml})$ in a volume of $35 \mathrm{ml} \mathrm{HBSS}$ with calcium and magnesium at $37^{\circ} \mathrm{C}$ with constant agitation for 1 hour. Digested cells were collected by centrifugation, resuspended in HBSS, and filtered through a $70-\mu \mathrm{m}$ and then a $40-\mu \mathrm{m}$ filter. The final pellet was resuspended in skeletal muscle growth media (Lonza, CC-3161) supplemented with 1× Glutamax (Cellgro, 25-015-CI) and $1 \times$ Primacin (InvivoGen, ant-pm-1) and preplated for 1 to 2 hours on non-collagen-coated flasks. Nonadherent cells were transferred to collagen-coated flasks with media supplemented with $10 \%$ FBS. Media were refreshed every 2-3 days and cells were frozen at $75 \%$ confluence. Oxygen consumption was measured using an XF24 Extracellular Flux Analyzer (Seahorse Biosciences) as previously described (77). Briefly, myoblasts were cultured to confluence (24-well plates) and then differentiated to myotubes in Dulbecco's modified Eagle's medium (DMEM)/5\% horse serum for 5 to 7 days before experimentation. Measurements of oxygen consumption were made continuously (every 10 seconds) while cells were sequentially treated with oligomycin, FCCP, and rotenone/myxothiazol.

Metabolomics sample preparation and analysis. Fetal gastroc samples were extracted in a solution of methanol, acetonitrile, and water $(5: 3: 2)$ at a concentration of $15 \mathrm{mg} / \mathrm{ml}$. For quantification of acylcarnitines, deuterated standards were included in the extraction solution according to the manufacturer's instructions (NSK-B, Cambridge Isotope Laboratories). Suspensions were vortexed, insoluble material removed, and supernatants isolated for metabolomics analysis by Ultra HPLC (UHPLC)-mass spectrometry as previously described $(78,79)$ using a Vanquish UHPLC coupled online to a Q Exactive mass spectrometer (Thermo Fisher). Extracts $(10 \mu \mathrm{l})$ were resolved in a Kinetex C18 column $(150 \times 2.1 \mathrm{~mm}$ i.d., 1.7- $\mu \mathrm{m}$ particle size; Phenomenex) using a 3-minute isocratic gradient at $250 \mu \mathrm{l} / \mathrm{minute}$ (mobile phase: $5 \%$ acetonitrile, $95 \% 18 \mathrm{MOhm} \mathrm{H}_{2} \mathrm{O}, 0.1 \%$ formic acid) or a 9 -minute gradient ( $5 \% \mathrm{~B}$ for the first 2 minutes, $5 \%-95 \%$ B over 1 minute, hold at $95 \%$ for 2 minutes, $95 \%-5 \%$ B over 1 minute, re-equilibrate for 3 minutes). Calibration was performed before each analysis using Pierce positive or negative ion mode calibration mixes (Thermo Fisher) to ensure sub-ppm error on the intact mass. Quality control was performed via the assessment of a technical mix injected after every 10 samples, as well as by comparison of internal standards included in every sample. Metabolite assignments were performed with the software MAVEN (80), upon conversion of .raw files into the .mzXML format using MassMatrix (www. massmatrix.net). Assignments were further confirmed by chemical formula determination from isotopic patterns, accurate intact mass, and retention time comparison against an in-house standard library (Sigma-Aldrich, IROATech). A heat map was created in gplot using R software version 3.1.2 following standard score ( $Z$ score) calculation of metabolite peak areas.

Statistics. Individual sample data points and the mean \pm SEM are graphed with the number of samples per group noted in the figure legends. For multiple comparisons, significant differences were analyzed by a 1- or 2-way ANOVA with a post-hoc analysis. Data containing only 2 groups were analyzed by an unpaired Student's $t$ test (Figure 6). The level of statistical significance was set at $P<0.05$. All analysis and graphs were made using Prism 7.0 software (GraphPad).

Study approval. All animal procedures were approved by and conducted in accordance with the Institutional Animal Care and Use Committee of the Oregon National Primate Research Center (ONPRC) and Oregon Health and Science University. The ONPRC abides by the Animal Welfare Act and Regulations enforced by the USDA and the Public Health Service Policy on Humane Care and Use of Laboratory Animals in accordance with the Guide for the Care and Use of Laboratory Animals published by the NIH. 


\section{Author contributions}

CEM, JEF, KLG, and KMA conceived of and designed the study. CEM, JAH, BH, AJC, SK, ML, AP, TN, $\mathrm{KH}, \mathrm{BCB}, \mathrm{BGD}, \mathrm{ALH}$, and DLT executed and designed experiments. CEM, SS, BGD, AJC, TN, IG, and $\mathrm{BCB}$ interpreted the results. CEM, SS, JEF, BH, AJC, KMA, and KLG drafted and revised the manuscript. All authors reviewed the manuscript. CEM is the guarantor of this work and, as such, had full access to all the data in the study and takes responsibility for the integrity of the data and the accuracy of the data analysis.

\section{Acknowledgments}

This research was supported by NIH grants K12 HD057022 (to C.E.M) and R24 DK090964 (to K.L.G, J.E.F., and K.M.A.), and by UCSD-UCLA Diabetes Research Center grant P30 DK063491 (to A.L.H.). S.S. was supported by NIH grants HD050837, DK063491, and AR058878. A.J.C, M.L., and S.K. were supported by the American Heart Association grant 15GRNT23190000 and NIFA/AFRI research grant 2015-67017-23143 (both to A.J.C.). The authors also thank Karalee Baquero, Peter Blundell, and the veterinary staff of the Oregon National Primate Research Center for excellent assistance in the use and care of the animals in this study. The authors also thank Matthew Jackman and Peter R. Baker II (University of Colorado) and Anne Murphy (University of California, San Diego) for insightful discussions on mitochondrial methodology and data interpretation.

Address correspondence to: Carrie E. McCurdy, 122c Esslinger Hall, 1240 University of Oregon, Eugene, Oregon 97403, USA. Phone: 541.346.4325; E-mail: cmccurd5@uoregon.edu.

1. Kim SY, Dietz PM, England L, Morrow B, Callaghan WM. Trends in pre-pregnancy obesity in nine states, 1993-2003. Obesity (Silver Spring). 2007;15(4):986-993.

2. Flegal KM, Carroll MD, Kit BK, Ogden CL. Prevalence of obesity and trends in the distribution of body mass index among US adults, 1999-2010. JAMA. 2012;307(5):491-497.

3. Dabelea D, Crume T. Maternal environment and the transgenerational cycle of obesity and diabetes. Diabetes. 2011;60(7):1849-1855.

4. Penfold NC, Ozanne SE. Developmental programming by maternal obesity in 2015: Outcomes, mechanisms, and potential interventions. Horm Behav. 2015;76:143-152.

5. Whitaker RC. Predicting preschooler obesity at birth: the role of maternal obesity in early pregnancy. Pediatrics. 2004;114(1):e29-e36.

6. Boney CM, Verma A, Tucker R, Vohr BR. Metabolic syndrome in childhood: association with birth weight, maternal obesity, and gestational diabetes mellitus. Pediatrics. 2005;115(3):e290-e296.

7. Li CC, et al. Maternal obesity and diabetes induces latent metabolic defects and widespread epigenetic changes in isogenic mice. Epigenetics. 2013;8(6):602-611

8. Nathanielsz PW, et al. Maternal obesity disrupts the methionine cycle in baboon pregnancy. Physiol Rep. 2015;3(11):11.

9. Suter M, et al. Epigenomics: maternal high-fat diet exposure in utero disrupts peripheral circadian gene expression in nonhuman primates. FASEB J. 2011;25(2):714-726.

10. Heerwagen MJ, Miller MR, Barbour LA, Friedman JE. Maternal obesity and fetal metabolic programming: a fertile epigenetic soil. Am J Physiol Regul Integr Comp Physiol. 2010;299(3):R711-R722.

11. Barrès R, Zierath JR. The role of diet and exercise in the transgenerational epigenetic landscape of T2DM. Nat Rev Endocrinol. 2016;12(8):441-451.

12. DeFronzo RA, Tripathy D. Skeletal muscle insulin resistance is the primary defect in type 2 diabetes. Diabetes Care. 2009;32 Supp1 2:S157-S163.

13. Anderson EJ, et al. Mitochondrial $\mathrm{H}_{2} \mathrm{O}_{2}$ emission and cellular redox state link excess fat intake to insulin resistance in both rodents and humans. J Clin Invest. 2009;119(3):573-581.

14. Rahimi $Y$, et al. Genetic activation of pyruvate dehydrogenase alters oxidative substrate selection to induce skeletal muscle insulin resistance. Proc Natl Acad Sci U S A. 2014;111(46):16508-16513.

15. Griffin $M E$, et al. Free fatty acid-induced insulin resistance is associated with activation of protein kinase $C$ theta and alterations in the insulin signaling cascade. Diabetes. 1999;48(6):1270-1274.

16. Koves TR, et al. Mitochondrial overload and incomplete fatty acid oxidation contribute to skeletal muscle insulin resistance. Cell Metab. 2008;7(1):45-56.

17. Boyle KE, Zheng D, Anderson EJ, Neufer PD, Houmard JA. Mitochondrial lipid oxidation is impaired in cultured myotubes from obese humans. Int J Obes (Lond). 2012;36(8):1025-1031.

18. DeBalsi KL, et al. Targeted metabolomics connects thioredoxin-interacting protein (TXNIP) to mitochondrial fuel selection and regulation of specific oxidoreductase enzymes in skeletal muscle. J Biol Chem. 2014;289(12):8106-8120.

19. Muoio DM, Neufer PD. Lipid-induced mitochondrial stress and insulin action in muscle. Cell Metab. 2012;15(5):595-605.

20. Seiler SE, et al. Obesity and lipid stress inhibit carnitine acetyltransferase activity. J Lipid Res. 2014;55(4):635-644 .

21. Muoio DM, et al. Muscle-specific deletion of carnitine acetyltransferase compromises glucose tolerance and metabolic flexibility. Cell Metab. 2012;15(5):764-777.

22. Schenk S, Harber MP, Shrivastava CR, Burant CF, Horowitz JF. Improved insulin sensitivity after weight loss and exercise training is mediated by a reduction in plasma fatty acid mobilization, not enhanced oxidative capacity. J Physiol (Lond). 
2009;587(Pt 20):4949-4961.

23. Affourtit C. Mitochondrial involvement in skeletal muscle insulin resistance: A case of imbalanced bioenergetics. Biochim Biophys Acta. 2016;1857(10):1678-1693.

24. Morino K, et al. Reduced mitochondrial density and increased IRS-1 serine phosphorylation in muscle of insulin-resistant offspring of type 2 diabetic parents. J Clin Invest. 2005;115(12):3587-3593.

25. Petersen KF, Dufour S, Befroy D, Garcia R, Shulman GI. Impaired mitochondrial activity in the insulin-resistant offspring of patients with type 2 diabetes. N Engl J Med. 2004;350(7):664-671.

26. Petersen KF, Dufour S, Shulman GI. Decreased insulin-stimulated ATP synthesis and phosphate transport in muscle of insulinresistant offspring of type 2 diabetic parents. PLoS Med. 2005;2(9):e233.

27. Petersen KF, Dufour S, Morino K, Yoo PS, Cline GW, Shulman GI. Reversal of muscle insulin resistance by weight reduction in young, lean, insulin-resistant offspring of parents with type 2 diabetes. Proc Natl Acad Sci U S A. 2012;109(21):8236-8240.

28. Befroy DE, et al. Impaired mitochondrial substrate oxidation in muscle of insulin-resistant offspring of type 2 diabetic patients. Diabetes. 2007;56(5):1376-1381.

29. Latouche $C$, et al. Maternal overnutrition programs changes in the expression of skeletal muscle genes that are associated with insulin resistance and defects of oxidative phosphorylation in adult male rat offspring. J Nutr. 2014;144(3):237-244.

30. Saben JL, et al. Maternal metabolic syndrome programs mitochondrial dysfunction via germline changes across three generations. Cell Rep. 2016;16(1):1-8.

31. Borengasser SJ, Faske J, Kang P, Blackburn ML, Badger TM, Shankar K. In utero exposure to prepregnancy maternal obesity and postweaning high-fat diet impair regulators of mitochondrial dynamics in rat placenta and offspring. Physiol Genomics. 2014;46(23):841-850.

32. Leary C, Leese HJ, Sturmey RG. Human embryos from overweight and obese women display phenotypic and metabolic abnormalities. Hum Reprod. 2015;30(1):122-132.

33. Igosheva N, et al. Maternal diet-induced obesity alters mitochondrial activity and redox status in mouse oocytes and zygotes PLoS One. 2010;5(4):e10074.

34. Wu LL, et al. Mitochondrial dysfunction in oocytes of obese mothers: transmission to offspring and reversal by pharmacological endoplasmic reticulum stress inhibitors. Development. 2015;142(4):681-691.

35. McCurdy CE, et al. Maternal high-fat diet triggers lipotoxicity in the fetal livers of nonhuman primates. J Clin Invest. 2009;119(2):323-335.

36. Frias AE, et al. Maternal high-fat diet disturbs uteroplacental hemodynamics and increases the frequency of stillbirth in a nonhuman primate model of excess nutrition. Endocrinology. 2011;152(6):2456-2464.

37. Scarpulla RC, Vega RB, Kelly DP. Transcriptional integration of mitochondrial biogenesis. Trends Endocrinol Metab. 2012;23(9):459-466

38. Samec S, Seydoux J, Dulloo AG. Role of UCP homologues in skeletal muscles and brown adipose tissue: mediators of thermogenesis or regulators of lipids as fuel substrate? FASEB J. 1998;12(9):715-724.

39. Kukat A, et al. Loss of UCP2 attenuates mitochondrial dysfunction without altering ROS production and uncoupling activity. PLoS Genet. 2014;10(6):e1004385.

40. Xia J, Wishart DS. MetPA: a web-based metabolomics tool for pathway analysis and visualization. Bioinformatics. 2010;26(18):2342-2344.

41. Comstock SM, et al. High-fat diet consumption during pregnancy and the early post-natal period leads to decreased $\alpha$ cell plasticity in the nonhuman primate. Mol Metab. 2012;2(1):10-22.

42. Rivera HM, et al. Maternal high-fat diet and obesity impact palatable food intake and dopamine signaling in nonhuman primate offspring. Obesity (Silver Spring). 2015;23(11):2157-2164.

43. Sullivan EL, Smith MS, Grove KL. Perinatal exposure to high-fat diet programs energy balance, metabolism and behavior in adulthood. Neuroendocrinology. 2011;93(1):1-8.

44. Suter MA, et al. Maternal high-fat diet modulates the fetal thyroid axis and thyroid gene expression in a nonhuman primate model. Mol Endocrinol. 2012;26(12):2071-2080.

45. Muoio DM. Metabolic inflexibility: when mitochondrial indecision leads to metabolic gridlock. Cell. 2014;159(6):1253-1262.

46. Kelley DE. Skeletal muscle fat oxidation: timing and flexibility are everything. J Clin Invest. 2005;115(7):1699-1702.

47. Gemma C, et al. Mitochondrial DNA depletion in small- and large-for-gestational-age newborns. Obesity (Silver Spring). 2006;14(12):2193-2199.

48. Sugden MC, Kraus A, Harris RA, Holness MJ. Fibre-type specific modification of the activity and regulation of skeletal muscle pyruvate dehydrogenase kinase (PDK) by prolonged starvation and refeeding is associated with targeted regulation of PDK isoenzyme 4 expression. Biochem J. 2000;346 Pt 3:651-657.

49. Buck MJ, Squire TL, Andrews MT. Coordinate expression of the PDK4 gene: a means of regulating fuel selection in a hibernating mammal. Physiol Genomics. 2002;8(1):5-13.

50. Brown LD, Rozance PJ, Bruce JL, Friedman JE, Hay WW, Wesolowski SR. Limited capacity for glucose oxidation in fetal sheep with intrauterine growth restriction. Am J Physiol Regul Integr Comp Physiol. 2015;309(8):R920-R928.

51. Garcia-Roves P, et al. Raising plasma fatty acid concentration induces increased biogenesis of mitochondria in skeletal muscle Proc Natl Acad Sci U S A. 2007;104(25):10709-10713.

52. Schenk S, Horowitz JF. Coimmunoprecipitation of FAT/CD36 and CPT I in skeletal muscle increases proportionally with fat oxidation after endurance exercise training. Am J Physiol Endocrinol Metab. 2006;291(2):E254-E260.

53. Maples JM, et al. Differential epigenetic and transcriptional response of the skeletal muscle carnitine palmitoyltransferase 1B (CPT1B) gene to lipid exposure with obesity. Am J Physiol Endocrinol Metab. 2015;309(4):E345-E356.

54. Kim JY, Hickner RC, Cortright RL, Dohm GL, Houmard JA. Lipid oxidation is reduced in obese human skeletal muscle. Am J Physiol Endocrinol Metab. 2000;279(5):E1039-E1044.

55. Davis JM, Auten RL. Maturation of the antioxidant system and the effects on preterm birth. Semin Fetal Neonatal Med. 2010;15(4):191-195

56. Nassi N, Ponziani V, Becatti M, Galvan P, Donzelli G. Anti-oxidant enzymes and related elements in term and preterm new- 
borns. Pediatr Int. 2009;51(2):183-187.

57. Malti N, et al. Oxidative stress and maternal obesity: feto-placental unit interaction. Placenta. 2014;35(6):411-416

58. Bell EL, Guarente L. The SirT3 divining rod points to oxidative stress. Mol Cell. 2011;42(5):561-568.

59. Kong X, et al. Sirtuin 3, a new target of PGC-1alpha, plays an important role in the suppression of ROS and mitochondrial biogenesis. PLoS ONE. 2010;5(7):e11707.

60. Echtay KS, et al. Superoxide activates mitochondrial uncoupling proteins. Nature. 2002;415(6867):96-99.

61. Anderson EJ, Yamazaki H, Neufer PD. Induction of endogenous uncoupling protein 3 suppresses mitochondrial oxidant emission during fatty acid-supported respiration. J Biol Chem. 2007;282(43):31257-31266.

62. Brand MD, et al. Oxidative damage and phospholipid fatty acyl composition in skeletal muscle mitochondria from mice underexpressing or overexpressing uncoupling protein 3. Biochem J. 2002;368(Pt 2):597-603.

63. Anedda A, López-Bernardo E, Acosta-Iborra B, Saadeh Suleiman M, Landázuri MO, Cadenas S. The transcription factor Nrf2 promotes survival by enhancing the expression of uncoupling protein 3 under conditions of oxidative stress. Free Radic Biol Med. 2013;61:395-407.

64. Vozza A, et al. UCP2 transports C4 metabolites out of mitochondria, regulating glucose and glutamine oxidation. Proc Natl Acad Sci U S A. 2014;111(3):960-965.

65. Barker DJ, Thornburg KL. Placental programming of chronic diseases, cancer and lifespan: a review. Placenta. 2013;34(10):841-845

66. Alwasel SH, et al. Sex differences in regional specialisation across the placental surface. Placenta. 2014;35(6):365-369.

67. Suter MA, et al. A maternal high-fat diet modulates fetal SIRT1 histone and protein deacetylase activity in nonhuman primates. FASEB J. 2012;26(12):5106-5114.

68. Grant WF, Nicol LE, Thorn SR, Grove KL, Friedman JE, Marks DL. Perinatal exposure to a high-fat diet is associated with reduced hepatic sympathetic innervation in one-year old male Japanese macaques. PLoS One. 2012;7(10):e48119.

69. Pound LD, Comstock SM, Grove KL. Consumption of a Western-style diet during pregnancy impairs offspring islet vascularization in a Japanese macaque model. Am J Physiol Endocrinol Metab. 2014;307(1):E115-E123.

70. Summers L, Clingerman KJ, Yang X. Validation of a body condition scoring system in rhesus macaques (Macaca mulatta): assessment of body composition by using dual-energy X-ray absorptiometry. J Am Assoc Lab Anim Sci. 2012;51(1):88-93

71. Spinazzi M, Casarin A, Pertegato V, Ermani M, Salviati L, Angelini C. Optimization of respiratory chain enzymatic assays in muscle for the diagnosis of mitochondrial disorders. Mitochondrion. 2011;11(6):893-904.

72. Spinazzi M, Casarin A, Pertegato V, Salviati L, Angelini C. Assessment of mitochondrial respiratory chain enzymatic activities on tissues and cultured cells. Nat Protoc. 2012;7(6):1235-1246.

73. Bergman BC, Perreault L, Hunerdosse DM, Koehler MC, Samek AM, Eckel RH. Intramuscular lipid metabolism in the insulin resistance of smoking. Diabetes. 2009;58(10):2220-2227.

74. Chicco AJ, et al. High fatty acid oxidation capacity and phosphorylation control despite elevated leak and reduced respiratory capacity in northern elephant seal muscle mitochondria. J Exp Biol. 2014;217(Pt 16):2947-2955.

75. Gnaiger E. Capacity of oxidative phosphorylation in human skeletal muscle: new perspectives of mitochondrial physiology. Int J Biochem Cell Biol. 2009;41(10):1837-1845.

76. Stewart JD, et al. Characterization of proliferating human skeletal muscle-derived cells in vitro: differential modulation of myoblast markers by TGF-beta2. J Cell Physiol. 2003;196(1):70-78.

77. Drew BG, et al. HSP72 is a mitochondrial stress sensor critical for Parkin action, oxidative metabolism, and insulin sensitivity in skeletal muscle. Diabetes. 2014;63(5):1488-1505.

78. Nemkov T, D’Alessandro A, Hansen KC. Three-minute method for amino acid analysis by UHPLC and high-resolution quadrupole orbitrap mass spectrometry. Amino Acids. 2015;47(11):2345-2357.

79. Sun K, et al. Sphingosine-1-phosphate promotes erythrocyte glycolysis and oxygen release for adaptation to high-altitude hypoxia. Nat Commun. 2016;7:12086.

80. Melamud E, Vastag L, Rabinowitz JD. Metabolomic analysis and visualization engine for LC-MS data. Anal Chem. 2010;82(23):9818-9826. 